\title{
Labelled Natural Deduction for Substructural Logics
}

\author{
KRYSIA BRODA, Department of Computing, Imperial College, \\ 180 Queen's Gate, London SWr 2BZ, UK. E-mail: kb@doc.ic.ac.uk
}

MARCELO FINGER, Departamento de Ciência da Computação, Instituto de Matemática e Estatística, Universidade de São Paulo, Brazil.E-mail: mfinger@ime.usp.br

ALESSANDRA RUSSO, Department of Computing, Imperial College, 180 Queen's Gate, London SW72BZ, UK.E-mail: ar3@doc.ic.ac.uk

\begin{abstract}
In this paper a uniform methodology to perform natural deduction over the family of linear, relevance and intuitionistic logics is proposed. The methodology follows the Labelled Deductive Systems (LDS) discipline, where the deductive process manipulates declarative units - formulas labelled according to a labelling algebra. In the system described here, labels are either ground terms or variables of a given labelling language and inference rules manipulate formulas and labels simultaneously, generating (whenever necessary) constraints on the labels used in the rules. A set of natural deduction style inference rules is given, and the notion of a derivation is defined which associates a labelled natural deduction style "structural derivation" with a set of generated constraints. Algorithmic procedures, based on a technique called resource abduction, are defined to solve the constraints generated within a structural derivation, and their termination conditions discussed. A natural deduction derivation is then defined to be correct with respect to a given substructural logic, if, under the condition that the algorithmic procedures terminate, the associated set of constraints is satisfied with respect to the underlying labelling algebra. Finally, soundness and completeness of the natural deduction system

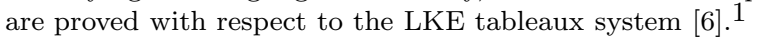

Keywords: Labelled Deductive Systems, Natural Deduction, Substructural Logics.

\section{Introduction}

This paper builds upon the methodology of Labelled Deductive Systems [ip] to develop a uniform and abductive natural deduction system for the family of linear, relevance and intuitionistic logics. The system is uniform in the sense that the set of labelled natural deduction rules is the same for all the three logics under consideration, and abductive in that it incorporates a technique for identifying, when possible, additional assumptions which can be used to detect, from the given derivation, theorems of the given substructural logic.

It is widely believed that natural deduction style proof theory is the only formal approach which comes as close as possible to informal rules of reasoning used in everyday

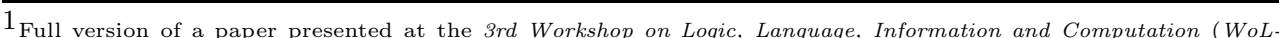
LIC'96), May 8-10, Salvador (Bahia), Brazil, organised by Univ. Federal de Pernambuco (UFPE) and Univ. Federal da Bahia (UFBA), and sponsored by IGPL, FoLLI, and ASL.

L. J. of the IGPL, Vol. 7 No. 3, pp. 
discourse ${ }^{2}$ ! . Despite this, in the field of substructural logics little research has been so far devoted to defining proof strategies based on natural deduction, and most work on automated theorem proving has concentrated mainly on sequent calculi [is]. The work in $[\overline{1} \overline{1}]$ is one of the few examples of a natural deduction system for substructural logic, which has been developed using the approach of generalised annotated logics. On the other hand, recent results have shown that Gabbay's methodology based on Labelled Deductive Systems (LDS) [99.] provides an ideal framework for developing uniform proof systems for various families of logics. Examples are [i6] where a uniform labelled semantic tableaux, called LKE system, is defined for a wide family of substructural logics, and [1] in which a uniform natural deduction style proof system, called MLDS, is described for a wide family of propositional and predicate modal logics. Furthermore, in [9. examples of labelled natural deduction rules for some substructural logics have also been described, which show some ways of handling labels to allow the same rules to be used in different substructural logics. However, this illustration covers only the implication fragment, no general soundness or completeness results for the rules are given, and moreover it does not include any algorithm for checking relationships between inferred labels. It is mainly given to illustrate a more general claim - LDS can be used to develop a uniform proof system for substructural logics. This paper substantiates this claim for the cases of linear, relevance and intuitionistic logic, also providing some initial results towards the development of automated labelled natural deduction theorem provers for substructural logics.

A uniform labelled natural deduction system for the family of linear, relevance and intuitionistic logics is given, whose set of inference rules is shared by each of these substructural logics. It is well known in the literature [i be uniquely defined in terms of a set of operational rules and a set of structural rules. The former are rules associated to each operator, whereas the latter are metarules which define how formulae can be used within a derivation. For example, in the case of relevance logic the permutation and contraction structural rules enforce assumptions to be used at least once but not necessarily in the order they are given. Results in the literature (see [i] for a detailed overview on substructural logics) show that the same set of operational rules is shared by the whole family of substructural logics and that it is each individual set of structural rules which uniquely identifies each logic by defining the properties of the associated consequence relation. Using these rules different theorems can be proved in different substructural logics, even though the basic operational rules are identical. For example, a formula of the form $A \rightarrow((A \otimes A \rightarrow B) \rightarrow B)$ can be proved to be a theorem of relevance logic but not proved to be a theorem of linear logic.

In Section 3 the three logics under consideration. These rules are defined on labelled formulae, called declarative units, and they perform the role of operational rules. Structural rules are instead implemented by different labelling algebras (uniquely associated with each individual logic), which define conditions on labels. A structural derivation is defined as a sequence of constrained declarative units which pair (inferred) labelled formulae with (possible empty) constraints on labels generated by the inference rules. The satisfiability of a generated set of constraints depends on the conditions of the

2 It is this closeness to the actual reasoning that had prompted Gentzen to put forward his natural deduction approach [H. 
underlying labelling algebra. The same set of constraints can be true with respect to one labelling algebra and false with respect to another. It is in this sense that the standard manipulation of assumptions given by the use of structural rules (see for instance Gentzen style calculus [i]l] should be seen in this system - to restrict and to control the use and the discharge of assumptions via the use of labels and of a labelling algebra, leaving unchanged the set of labelled natural deduction rules. The three different substructural logics are captured, therefore, by simply changing the underlying labelling algebra. This facilitates a uniform proof system whose derivation process is well structured and more human-oriented.

In this paper, attention is restricted to the substructural fragment containing the operators $\rightarrow$ and $\otimes$. Extension to the whole set of substructural operators, including the exponential operator, is currently under investigation. A first prototype theorem prover for full linear logic has been developed in [12]. In Section $2_{-1}^{1}$ language and syntax of the system are given together with the notion of a labelling algebra. The latter is defined in terms of a set of elements, a partial ordering relation and a binary operator. Three different types of labelling algebras are defined by imposing on the

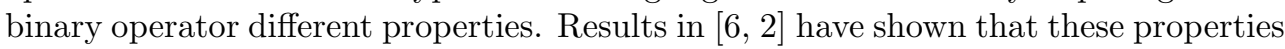
correspond to the structural rules of the substructural consequence relation. In the case of linear logic, the permutation property of the consequence relation is captured by requiring the binary operator of the labelling algebras to be commutative; for relevance logic, the binary operator satisfies commutativity and contraction, and for intuitionistic logic it satisfies also monotonicity. In Section $\overline{3}$, , a set of labelled natural deduction rules, together with the notion of a structural derivation, are defined, and an example is given to illustrate the uniform property of the system. These rules are based on those originally proposed by Prawitz for classical logic [14in. Algorithmic procedures are given (see Section $\left.\underline{4}_{1}^{\prime}\right)$, which allow the sets of constraints generated within derivations to be solved accordingly with the underlying labelling algebra. The termination property and scope of such algorithms are also discussed. It is these algorithms that are the main contribution of this paper - their simplicity is facilitated by the use of natural deduction. A structural derivation is then defined to be correct with respect to a given substructural logic, if, under the condition that the algorithmic procedure terminates, the set of constraints generated in the structural derivation is satisfied with respect to the labelling algebra associated with the underlying logic. In Section $\bar{p}_{v}^{\prime}$, the natural deduction system is shown to be sound and complete with respect to the LKE tableaux system [i, $\left.{ }^{6}\right]$. This consists of showing that (i) given a natural deduction derivation of a labelled formula with a satisfied set of constraints, there exists a closed LKE refutation of that labelled formula, and that (ii) the converse holds. Part (i) is proved in Section 5.2 by considering an equivalent extended LKE system, whereas part (ii) is briefly discussed in Section 15.1, as already shown in [2: The paper concludes with some final discussion and comparisons.

Finally, some remarks regarding syntactic notations. Throughout the paper lowercase letters from $u$ to $z$ are used to refer to terms in the system, whereas upper-case letters denote wffs of the system. Integer subscripts may also be used with any of these letters. Each of the three substructural logics considered in this paper will sometimes be referred to as LL for linear logic, RL for relevance logic and IL for intuitionistic logic. 


\section{Labelling Algebras for Substructural Logics}

In this section basic definitions of the language and syntax of the system are given, together with the notion of a labelling algebra and the meaning of a declarative unit, for the substructural fragment $\{\rightarrow, \otimes\}$.

The language of the system is defined as an ordered pair $\left\langle L_{\{\rightarrow, \otimes\}}, L_{L}\right\rangle$, where $L_{L}$ is a labelling language and $L_{\{\rightarrow, \otimes\}}$ is a standard propositional substructural language restricted to the set of operators $\{\rightarrow, \otimes\}$. Wffs of $L_{\{\rightarrow, \otimes\}}$ are defined in the standard way. The labelling language $L_{L}$ is composed of the constant symbol 1 , a countable set of symbols $\left\{a, b, \ldots, a_{1}, b_{1}, \ldots\right\}$ called solo parameters, a countable set of variables $\left\{\gamma, \delta, \gamma_{1}, \delta_{1}, \gamma_{2}, \delta_{2}, \ldots\right\}$, a binary function symbol $\circ$ and a binary relation $\sqsubseteq$. Terms of the labelling language are defined inductively as consisting of 1 , solo parameters and variables, together with expressions of the form $x \circ y$, where $x$ and $y$ are terms. Terms of $L_{L}$ are generally referred to as labels, the term 1 and the solo parameters are also called atomic labels, whereas these and any other ground term are often referred to as ground labels. The syntax of the system is given by two different types of syntactic entity, the declarative unit and the label constraint. A declarative unit is defined as a pair of the form formula : label, expressing that a formula is true relative to a piece of information (i.e. label). The formula component is written in $L_{\{\rightarrow, \otimes\}}$ and the label component is a term of $L_{L}$. A label constraint in the language $L_{L}$ is of the form $x \sqsubseteq y$ where $x$ and $y$ are labels.

Both labels and the relation $\sqsubseteq$ are interpreted onto a labelling algebra, given in Definition 2.1 . Atomic labels are interpreted in a labelling algebra as themselves in the style of an Herbrand interpretation. Informally, labels are interpreted as "pieces of information" relative to which formulae are evaluated true or false. The atomic labels, given by the solo parameters of $L_{L}$, are used to name particular pieces of information. The binary relation $\sqsubseteq$ behaves as a kind of Kripke-style accessibility relation between pieces of information, according to which, if a piece of information $y$ is accessible from a piece of information $x$, then $y$ verifies all the formulae which are verified at $x$ (if any). For simplicity, the term "label" will sometimes be used to refer to its interpretation in the algebra, and the same notations are used between the terms of the labelling language and the elements of the labelling algebra, as well as between the binary relation of $L_{L}$ and the partial ordering of the algebra.

Definition 2.1 $A$ labelling algebra $\mathcal{L}_{A}$ is a tuple $(\mathcal{L}, \circ, 1, \sqsubseteq)$ such that:

1. $\mathcal{L}$ is a set of elements, where $1 \in \mathcal{L}$;

2. $\sqsubseteq$ is a preordering;

3. $\circ$ is a binary operation on $\mathcal{L}$, which satisfies the following properties:

(a) associativity: $x \circ(y \circ z) \sqsubseteq(x \circ y) \circ z$ and $(x \circ y) \circ z \sqsubseteq x \circ(y \circ z)$;

(b) identity: for every $x \in \mathcal{L}, x \sqsubseteq x \circ 1 \sqsubseteq 1 \circ x$ and $1 \circ x \sqsubseteq x \circ 1 \sqsubseteq x$;

(c) order preserving: for every $x, y \in \mathcal{L}$, if $x \sqsubseteq y$ then $x \circ z \sqsubseteq y \circ z$ and $z \circ x \sqsubseteq z \circ y$ for every $z \in \mathcal{L}$;

The elements of a labelling algebra are pieces of information, or resources, used to verify formulae. The o operator allows concatenation of resources. In general, formulae which are verified by means of individual resources are not necessarily verified by the resource composition of the individual ones. The associativity property of the o operator means that composed elements of the algebra, which differ only in the 
way their components are associated, are identical pieces of information. Adopting the view of a labelling algebra as a "structure" of resources $\left[\begin{array}{l}\overline{6}_{0}^{6} \\ i 2\end{array} \overline{2}\right.$, the associativity property of o implies that the composition over the same "sequence" of resources is arbitrary. This implies that if a formula is verified by means of a composition of resources of the form $x \circ(y \circ z)$, then the same formula can be verified composing the same list of resources in a different way.

In addition to the basic properties given in Definition $\overline{2} . \overline{1}$, the binary operator o may satisfy other properties, so defining different types of labelling algebras. In this paper the following properties are considered:

$$
\begin{array}{ll}
\text { commutativity: } & x \circ y \sqsubseteq y \circ x \\
\text { contraction: } & x \circ x \sqsubseteq x \\
\text { monotonicity: } & x \sqsubseteq x \circ y
\end{array}
$$

The commutativity property states that if a formula is verified by means of a given sequence of resources, then it can be verified changing the order of the resources in the sequence. The contraction property instead guarantees that if a formula is verified using more than one occurrence of a given resource, then it can be verified using a fewer number of occurrences. Finally, the monotonicity property gives that if a formula is verified by means of a given resource, then it is still verified if this resource is combined with any other resource. Considering each atomic resource as uniquely associated to each formula, a sequence of resources can be read as a sequence of formulae. Under this interpretation, it is possible to see a correspondence between the above three properties of the o operator and the properties of the substructural consequence relation. In $\left[\begin{array}{l}1 \\ 0\end{array}\right.$ that the commutativity property corresponds to the permutation rule, the contraction property to the contraction rule and the monotonicity property to the weakening rule. On the basis of this correspondence it is possible to show that different labelling algebras identify different substructural logics (see [2in] for further details). In this paper, three types of labelling algebras are considered. These are respectively the labelling algebra whose operator $\circ$ is commutative, the labelling algebra whose operator $\circ$ satisfies commutativity and contraction, and the labelling algebra whose operator $\circ$ satisfies commutativity, contraction and monotonicity. The first corresponds to Girard's Linear Logic (LL) [1] ${ }_{1}^{1}$, the second to Relevance Logic (RL) and the third to Intuitionistic Logic. This is summarised in Table ${ }_{1}^{\prime}$, where the notation $\mathcal{L}_{\Delta}$, with $\Delta \in\{\mathrm{LL}, \mathrm{RL}, \mathrm{IL}\}$, is also introduced. Note that, throughout the paper, the notation $\mathcal{L}_{\Delta}^{S}$, called the labelling algebra associated with $S$, is instead used to denote the particular labelling algebra $\mathcal{L}_{\Delta}$ whose set of elements $\mathcal{L}$ is equal to a given set $S$.

The meaning of a declarative unit is defined in terms of a valuation function.

Definition 2.2 Let $\mathcal{L}_{A}=(\mathcal{L}, \circ, 1, \sqsubseteq)$ be a labelling algebra and let $\mathbf{F}$ be the set of wffs of $L_{\{\rightarrow, \otimes\}}$. A valuation over $\mathcal{L}_{A}$ is a mapping $V: \mathbf{F} \times \mathcal{L} \longrightarrow\{T, F\}$ satisfying the following conditions:

1. For all formulae $A$, if $V(A, x)=T$ and $x \sqsubseteq y$, then $V(A, y)=T$.

2. For all formulae $A$, if $V(A, x)=T$ for some $x \in \mathcal{L}$, then there exists also $a \in \mathcal{L}$, called the $A$-characteristic, where $V(A, a)=T$ and $a$ is the least such element with respect to the ordering $\sqsubseteq$. That is, for any $y \in \mathcal{L}$, if $V(A, y)=T$ then $a \sqsubseteq y$.

3. For each wff of the form $A \rightarrow B$ and for each label $x$ $V(A \rightarrow B, x)=T \Leftrightarrow \forall y[V(A, y)=T$ implies $V(B, x \circ y)=T]$. 


\begin{tabular}{|c|c|}
\hline Conditions on $\sqsubseteq$ & Labelling algebras \\
\hline$\{x \circ y \sqsubseteq y \circ x\}$ & $\mathcal{L}_{\mathrm{LL}}$ \\
$\{x \circ y \sqsubseteq y \circ x$, & \\
$x \circ x \sqsubseteq x\}$ & $\mathcal{L}_{\mathrm{RL}}$ \\
$\{x \circ y \sqsubseteq y \circ x$, & \\
$x \circ x \sqsubseteq x$, & \\
$x \sqsubseteq x \circ y\}$ & $\mathcal{L}_{\mathrm{IL}}$ \\
\hline
\end{tabular}

TABLE 1. Classes of labelling algebras

\section{For each wff of the form $A \otimes B$ and for each label $x$ \\ $V(A \otimes B, x)=T \Leftrightarrow \exists y, z[y \circ z \sqsubseteq x$ and $V(A, y)=T$ and $V(B, z)=T]$.}

In the above definition condition (1) expresses the "hereditary" property of the truth values with respect to the partial ordering $\sqsubseteq$, condition (2) plays an important role in the definition of some of the natural deduction rules, and in proving the correspondence of the natural deduction system with respect to the LKE system, whereas conditions (3) and (4) provide the semantic meaning of the two substructural operators $\rightarrow$ and $\otimes$, respectively.

Using the above notion of a valuation function, a declarative unit $A: x$ is said to be satisfied if and only if $V(A, x)=T$, where the argument $x$ of $V$ is the interpretation of the label $x$ in the labelling algebra. Given a labelling algebra $\mathcal{L}_{\Delta}$, for some $\Delta \in$ $\{\mathrm{LL}, \mathrm{RL}, \mathrm{IL}\}$, and a valuation $V$ over $\mathcal{L}_{\Delta}$, the tuple $\left\langle\mathcal{L}_{A}, V\right\rangle$ can be seen as a semantic structure for the fragment $\{\rightarrow, \otimes\}$ of the substructural logic $\Delta$. A treatment of implication and (multiplicative) conjunction similar to that of conditions (3) and (4) in Definition 2.2 can be found in several places in the literature; in particular, in [1 30 , in the context of modal logics, the labels are seen as possible worlds and implication has the same behaviour as condition (3).

\section{A Uniform Natural Deduction System}

In this section a uniform labelled natural deduction style proof system is described for the fragment $\{\rightarrow, \otimes\}$ of linear, relevance and intuitionistic logics. The set of inference rules is defined together with the notions of a "structural derivation" and "label constraints". An example is also given which shows how the same set of inference rules can be used to construct structural derivations in any of the three substructural logics.

It is strongly believed that this system expands the study of the natural deduction formalism, initially developed by Gabbay in $[90$, showing how the naturalness and the closeness to actual reasoning, typical of natural deduction calculus, can also capture substructural deductive processes. There is in fact no reason for restricting the attention only to tableau methods and sequent calculi $\left[\begin{array}{l}6 \\ 6\end{array}\right.$ 
can be equally expressive.

Label constraints. Most of the labelled natural deduction rules, defined in this section, have associated constraints. These are called label constraints and are of the form $x \sqsubseteq y$, where $x$ and $y$ are labels in the language $L_{L}$. For any given constraint $x \sqsubseteq y$, the terms $x$ and $y$ will often be referred to as the left-hand side (LHS) and the right-hand side (RHS) of the constraint. Label constraints are of two different types: imposed constraints (ICs) and required constraints $(R C \mathrm{~s})$. Their satisfiability depends on the properties of the underlying labelling algebra. In Section $\overline{4}$ a basic algorithmic procedure is defined for solving such label constraints and extensions of this procedure are also given, which take into account the specific additional properties of each type of labelling algebra. The process of detecting whether a structural derivation is a derivation with respect to a given logic reduces to resolving the set of label constraints generated within the structural derivation. The algorithms use also a technique called resource abduction which, whenever they terminate, provide a way of "abducing" the additional assumptions that can be used to detect theorems of a given substructural logic.

Structural derivations are sequences of constrained declarative units that are either given by initial assumptions or generated by the application of natural deduction inference rules to earlier constrained declarative units in the sequence. A constrained declarative unit is given by a declarative unit and its "associated constraints" (which eventually need to be satisfied). For a declarative unit $A: x$, which is a consequence of a natural deduction rule, the associated constraints are of the form $\left\langle\Gamma_{A: x}, I C_{A: x}\right\rangle$. The component $\Gamma_{A: x}$ is a set of pairs of the form $\left\langle I C_{B: y}, R C_{B: y}\right\rangle$ for each declarative unit $B: y$ included in the sub-derivation(s) of the rule together with the new generated pair $\left\langle I C_{A: x}, R C_{A: x}\right\rangle^{3}$. The component $R C_{A: x}$ (if not empty) is a single label constraint associated with the rule. The set $\Gamma_{A: x}$ has therefore the role of accumulating constraints generated in the sub-derivations of the inference rules. This is because the solution of the label constraints takes place only at the end of a structural derivation, and the constraints generated within sub-derivations would otherwise be lost. The component $I C_{A: x}$ of the new generated constraint is instead a set of imposed constraints that accumulates the new imposed constraint (if any) generated by the rule and the imposed constraints generated in the structural derivation before the rule application. The process of accumulating imposed constraints is formally described in the definitions of the natural deduction rules.

Imposed constraints generated within a sub-derivation (or a box in the graphical representation) are used only within that sub-derivation. Associated constraints will sometimes be indexed with the meta-variable denoting the constrained declarative units to which they belong. For instance, given a constrained declarative unit $\alpha$, associated constraints included in $\alpha$ will be denoted by the pair $\left\langle\Gamma_{\alpha}, I C_{\alpha}\right\rangle . \Gamma_{\alpha}$ and/or $I C_{\alpha}$ may well be empty sets. For example, a constrained declarative unit $\alpha$ given by an initial assumption has its associated constraints given by the pair $\langle\emptyset, \emptyset\rangle$.

Definition 3.1 Let $T$ be a set of constrained declarative units and let $A: x$ be a declarative unit with the associated constraints $\left\langle\Gamma_{A: x}, I C_{A: x}\right\rangle$. Then, a structural derivation of $A: x$ from $T$ with associated constraints $\left\langle\Gamma_{A: x}, I C_{A: x}\right\rangle$ and domain $D$ is a sequence

3 Note that for a consequence $A: x$ of a natural deduction rule that does not have sub-derivations, the set $\Gamma_{A: x}$ is simply the singleton set $\left\{\left\langle I C_{A: x}, R C_{A: x}\right\rangle\right\}$. 
$T, \beta_{1}, \beta_{2}, \ldots, \beta_{n}$ such that

- $\beta_{i}$, for each $1 \leq i \leq n$, are constrained declarative units,

- $\beta_{n}$ is the constrained declarative unit given by the declarative unit $A: x$ and its associated constraints $\left\langle\Gamma_{A: x}, I C_{A: x}\right\rangle$,

- the declarative unit of each $\beta_{i}, 1 \leq i \leq n$, is the consequence of a natural deduction rule from the set of constrained declarative units $T \cup\left\{\beta_{1}, \ldots, \beta_{i-1}\right\}$, with associated constraints $\left\langle\Gamma_{\beta_{i}}, I C_{\beta_{i}}\right\rangle$.

- $D$ is the set of atomic labels that appear in the constraints.

It is obvious that the concatenation of natural deduction rules applications to construct a structural derivation reflects the standard natural deduction property that if there exists a structural derivation of a constrained declarative unit $\alpha$ from $T$ and there exists a structural derivation of a constrained declarative unit $\beta$ from $T \cup\{\alpha\}$, then there exists also a structural derivation of $\beta$ from $T$. The definition of each natural deduction rule is given below. Note that, for a given set of constrained declarative units $T$ of the form $\left\{\alpha_{1}, \ldots, \alpha_{n}\right\}$, and a declarative unit $A: x, A: x \in T$ will denote that there exists some $i, 1 \leq i \leq n$, such that $\alpha_{i}$ is the constrained declarative unit composed of the declarative unit $A: x$ and its associated constraints $\left\langle\Gamma_{\alpha_{i}}, I C_{\alpha_{i}}\right\rangle$.

Definition 3.2 Let $T$ be a set of constrained declarative units of the form $\left\{\alpha_{1}, \ldots, \alpha_{n}\right\}$. $A$ declarative unit of the form $B: x \circ y$ is a consequence of $(\rightarrow \mathcal{E})$ rule from the set $T$ with associated constraints $\left\langle\Gamma_{B: x \circ y}, I C_{B: x \circ y}\right\rangle$, if

- $A \rightarrow B: x \in T$,

- $A: y \in T$,

- $\Gamma_{B: x \circ y}=\left\{\left\langle I C_{B: x \circ y}, R C_{B: x \circ y}\right\rangle\right\}$, where $I C_{B: x \circ y}=I C_{\alpha_{n}}$ and $R C_{B: x \circ y}=\emptyset$.

Definition 3.3 Let $T$ be a set of constrained declarative units of the form $\left\{\alpha_{1}, \ldots, \alpha_{n}\right\}$. Let $\alpha_{n+1}$ be a constrained declarative unit composed of the assumption $A:$ a, where $a$ is a solo parameter, and the associated constraints $\left\langle\Gamma_{A: a}, I C_{A: a}\right\rangle=\left\langle\emptyset, I C_{\alpha_{n}}\right\rangle . A$ declarative unit of the form $A \rightarrow B: x$ is a consequence of $(\rightarrow \mathcal{I})$ rule from the set $T$ with associated constraints $\left\langle\Gamma_{A \rightarrow B: x}, I C_{A \rightarrow B: x}\right\rangle$, if

- there exists a structural derivation of $B: x \circ$ a from $T \cup\left\{\alpha_{n+1}\right\}$ with associated constraints $\left\langle\Gamma_{B: x \circ a}, I C_{B: x \circ a}\right\rangle$,

- $\Gamma_{A \rightarrow B: x}=\Gamma_{B: x \circ a} \cup\left\{\left\langle I C_{A \rightarrow B: x}, R C_{A \rightarrow B: x}\right\rangle\right\}$, where the new associated imposed constraint $I C_{A \rightarrow B: x}=I C_{\alpha_{n}}$ and required constraint $R C_{A \rightarrow B: x}=\emptyset$.

The introduction and elimination rules for the $\rightarrow$ operator together reflect the semantic interpretation of $\rightarrow$ given in Section $\overline{2}_{1}^{1}$ (see condition (3) of Definition $\overline{2} . \overline{2}$ ).

Definition 3.4 Let $T$ be a set of constrained declarative units of the form $\left\{\alpha_{1}, \ldots, \alpha_{n}\right\}$. Declarative units of the form $A: a$ and $B: b$, where $a$ and $b$ are solo parameters, are, individually, consequences of $(\otimes \mathcal{E})$ rule from the set $T$ with respective associated constraints $\left\langle\Gamma_{A: a}, I C_{A: a}\right\rangle$ and $\left\langle\Gamma_{B: b}, I C_{B: b}\right\rangle$, where $\Gamma_{A: a}=\Gamma_{B: b}$ and $I C_{A: a}=I C_{B: b}$, if

- $A \otimes B: x \in T$,

- $\Gamma_{A: a}=\left\{\left\langle I C_{A: a}, R C_{A: a}\right\rangle\right\}$, where $I C_{A: a}=I C_{\alpha_{n}} \cup\{a \circ b \sqsubseteq x\}$, and $R C_{A: a}=\emptyset$. 
Definition 3.5 Let $T$ be a set of constrained declarative units of the form $\left\{\alpha_{1}, \ldots, \alpha_{n}\right\}$. $A$ declarative unit of the form $A \otimes B: x$ is a consequence of $(\otimes \mathcal{I})$ rule from the set $T$ with associated constraints $\left\langle\Gamma_{A \otimes B: x}, I C_{A \otimes B: x}\right\rangle$, if

- there exists a structural derivation of $A: \gamma_{1}$ from $T$ with associated constraints $\left\langle\Gamma_{A: \gamma_{1}}, I C_{A: \gamma_{1}}\right\rangle$,

- there exists a structural derivation of $B: \gamma_{2}$ from $T$ with associated constraints $\left\langle\Gamma_{B: \gamma_{2}}, I C_{B: \gamma_{2}}\right\rangle$,

- $\Gamma_{A \otimes B: x}=\Gamma_{A: \gamma_{1}} \cup \Gamma_{B: \gamma_{2}} \cup\left\{\left\langle I C_{A \otimes B: x}, R C_{A \otimes B: x}\right\rangle\right\}$, where $I C_{A \otimes B: x}=I C_{\alpha_{n}}$ and $R C_{A \otimes B}=\left\{\gamma_{1} \circ \gamma_{2} \sqsubseteq x\right\}$.

For the operator $\otimes$, the introduction and elimination rules, validated by their satisfied constraints, together reflect the semantic interpretation of $\otimes$ given in Definition $\underline{2} . \overline{2}$ (see condition (4)). Specifically, the required constraint associated with the consequence of the $(\otimes \mathcal{I})$ rule corresponds to the condition on the labels given in the right-hand side of condition (4), whereas the atomic labels $a$ and $b$ used in the $(\otimes \mathcal{E})$ rule can be seen as "Skolem" constants.

Definition 3.6 Let $T$ be a set of constrained declarative units of the form $\left\{\alpha_{1}, \ldots, \alpha_{n}\right\}$ and let $A: x$ be a declarative unit. A declarative unit of the form $A: y$ is a consequence of the (Tick) rule from the set $T$ with associated constraints $\left\langle\Gamma_{A: y}, I C_{A: y}\right\rangle$, if

- $A: x \in T$,

- $\Gamma_{A: y}=\left\{\left\langle I C_{A: y}, R C_{A: y}\right\rangle\right\}$, where $I C_{A: y}=I C_{\alpha_{n}}$ and $R C_{A: y}=\{x \sqsubseteq y\}$.

The two introduction rules can be interpreted procedurally as follows: "to show $A \rightarrow B: x$, assume $A: a$ and show $B: x \circ a$ ", and "to show $A \otimes B: x$ show $A: \gamma_{1}$ and $B: \gamma_{2}$ ", where $\gamma_{1} \circ \gamma_{2} \sqsubseteq x$. They are both used in reasoning backwards (i.e. reasoning from the goal formula to be proven), whereas the two elimination rules are used forwards (i.e. reasoning from the given assumptions). Their use in backward reasoning explains the variable labels in the sub-goals of the $(\otimes \mathcal{I})$ and (Lemma) rules. For example, in the $(\otimes \mathcal{I})$ rule, to show $A \otimes B: x$, the sub-goals $A$ and $B$ need to be proved with some variable labels $\gamma_{1}$ and $\gamma_{2}$, respectively. Because of backwards reasoning it is not possible to know a priori the amount of resources needed to show these two formulae so that $A \otimes B$ can be proved at $x$. Forward reasoning within the two sub-proofs of this rule would lead eventually to the derivation of sub-goals $A$ and $B$ with particular ground labels. The application of the (Tick) rule would then close each of these sub-proofs and the required constraints would then facilitate the instantiation of the variable labels $\gamma_{1}$ and $\gamma_{2}$ with these specific ground labels. In fact, the (Tick) rule allows complete sub-proofs to be recognised, enabling forwards and backwards reasoning within a derivation to be "linked" together. From a semantic point of view, this rule, together with its satisfied required constraint, reflect the hereditary property of the valuation function (i.e. condition (1) of Definition 2.21 ).

Definition 3.7 Let $T$ be a set of constrained declarative units of the form $\left\{\alpha_{1}, \ldots, \alpha_{n}\right\}$. $A$ declarative unit of the form $A: \gamma$ is a consequence of the (Lemma) rule from the set $T$ with associated constraints $\left\langle\Gamma_{A: \gamma}, I C_{A: \gamma}\right\rangle$, if

- there exists a structural derivation of $A: \gamma$ from $T$ with associated constraints $\left\langle\Gamma_{A: \gamma}^{\prime}, I C_{A: \gamma}^{\prime}\right\rangle$, 
- the constraints associated with the consequence $A: \gamma$ are such that $\Gamma_{A: \gamma}=\Gamma_{A: \gamma}^{\prime} \cup$ $\left\{\left\langle I C_{A: \gamma}, R C_{A: \gamma}\right\rangle\right\}$, where $I C_{A: \gamma}=I C_{\alpha_{n}}$ and $R C_{A: \gamma}=\emptyset$.

The (Lemma) rule can be seen as a way of incorporating the notion of "cut" into the natural deduction system. It is used in a derivation whenever the operational rules cannot derive any new formula from a given set of constrained declarative units. The rule provides a way of introducing a new relevant formula not as an assumption but as a lemma whose proof is constructed in the sub-derivation of the rule itself. An argument similar to that given for the (Tick) rule explains the use of a variable label. An example of a derivation that requires this rule is that of proving $C: c$ from the assumption $(A \rightarrow A) \rightarrow C: c$ - the (Lemma) rule is needed because of the goal-oriented nature of the introduction rules. This rule also facilitates in Section an easier proof of the correspondence with the LKE system, since the latter includes the cut rule explicitly. An example derivation which uses the (Lemma) rule is given in Figure $\overline{1}_{1}^{1}$. Note that, within a structural derivation, solo parameters are used so that their atomic occurrences label only the formulae they are first introduced with, and appear repeatedly in the derivation whenever such formulae are re-introduced.

A graphical representation of these inference rules is given in Table $\overline{2}_{n}^{-1}$ adopting a presentation style introduced in [3i] for the definition of a classical natural deduction style proof system. Note that only the new generated imposed and required constraints are shown in Table $\overline{2}$. New imposed constraints are introduced by the $(\otimes \mathcal{E})$ rule (see the second part of condition (iii) shown in Table $\left.\mathbf{2}^{-i}\right)$. Their validity is "imposed" by the occurrences of the $\otimes$-formulae, consistently with the semantic interpretation of the $\otimes$ operator. In this type of constraint, variables only ever occur on the right-hand side. New required constraints are instead generated by the $(\otimes \mathcal{I})$ rule and by the (Tick) rule (respectively conditions (ii) and (iv) shown in Table 2 ?il).

In the $(\otimes \mathcal{I})$ and (Lemma) rules, boxes have mainly the function of separating sub-derivations and therefore of structuring the proof. The standard use of boxes also helps in keeping track of the "local" imposed constraints that need to be used for resolving label constraints introduced in the sub-derivation. It is only in the case of $(\rightarrow \mathcal{I})$ that the introduction of a box implies also the introduction of a new assumption and that its closure implies the discharge of that assumption. It is this structural property of the derivations that facilitates an easy search of solutions for the generated set of constraints. This is one of the main advantages of this system which can make it preferable to other proof systems (e.g., the LKE tableau system

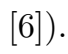

The notion of derivability. In this system the notion of derivability extends the standard notion of natural deduction derivability. A structural derivation does not guarantee itself the derivability of a formula in a given substructural logic. Arbitrary structural derivations could in fact be constructed, but only those whose set of associated constraints is satisfied in the underlying labelling algebra, are correct derivations in the underlying logic. This is captured by the following definitions.

Definition 3.8 Let $\Delta \in\{L L, R L, I L\}$, let $\left\{\left\langle I C_{1}, R C_{1}\right\rangle, \ldots,\left\langle I C_{n}, R C_{n}\right\rangle\right\}$ be a set of pairs of imposed and required constraints, let $\gamma_{1}, \ldots, \gamma_{k}$, with $k \geq 0$, and $D$ be, respectively, the variables and set of atomic labels that appear in the constraints. Let $\mathcal{L}_{\Delta}^{D}$ be the labelling algebra associated with $D$. Then the set of constraints $\left\{\left\langle I C_{1}, R C_{1}\right\rangle, \ldots,\left\langle I C_{n}, R C_{n}\right\rangle\right\}$ is satisfied in $\mathcal{L}_{\Delta}^{D}$ iff there exists a ground instantia- 
3. ${ }^{-} \bar{U} \bar{U} \bar{N} \bar{F} \bar{O} \bar{M} \bar{N} \bar{A} \bar{U} \bar{U} \bar{A} \bar{L} \bar{D} \bar{E} \bar{D} \bar{U} \bar{C} \bar{T} \bar{O} \bar{N} \bar{S} \bar{S} \bar{S} \bar{T} \bar{M} \bar{M}$

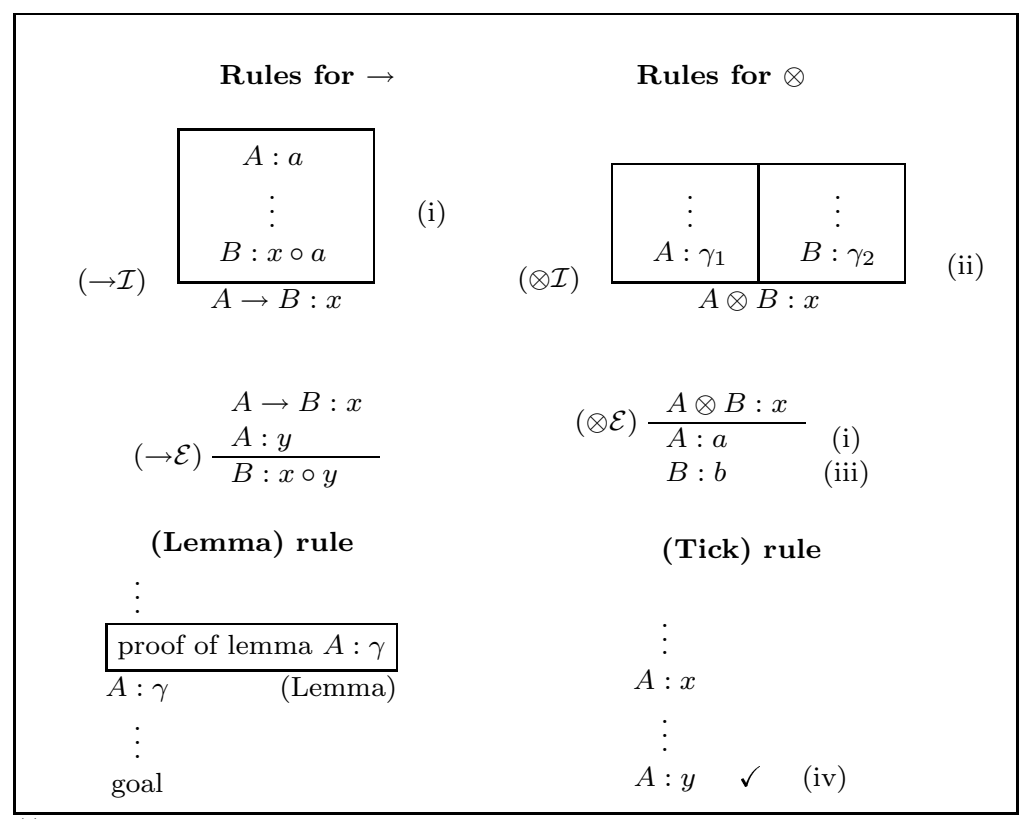

(i) $a$ is a solo parameter

(ii) $\gamma_{1} \circ \gamma_{2} \sqsubseteq x$

(iii) $b$ is a solo parameter, and $a \circ b \sqsubseteq x$

(iv) $x \sqsubseteq y$

TABLE 2. ND Rules for the substructural fragment $\{\rightarrow, \otimes\}$.

tion in $\mathcal{L}_{\Delta}^{D}$ of $\gamma_{1}, \ldots, \gamma_{k}$, called a solution, such that for each $1 \leq i \leq n, R C_{i}$ is true in $\mathcal{L}_{\Delta}^{D}$ extended with the related $I C_{i}$ imposed constraints.".

In Section "4i algorithmic procedures for "solving" a set of label constraints are described. The set of constraints associated with a structural derivation is defined on the basis of the constraints associated with each constrained declarative unit in the structural derivation.

Definition 3.9 Let $T, \alpha_{1}, \ldots, \alpha_{n}$ be a structural derivation of $A: x$ from the $\operatorname{set} T$ of initial assumptions. Let $\Gamma$ be the set of constraints given by $\Gamma=\bigcup_{1<i<n} \Gamma_{\alpha_{i}}$. The set of constraints associated with the structural derivation is the set $\bar{C}=\left\{\left\langle I C_{j}, R C_{j}\right\rangle \mid\right.$ $\left\langle I C_{j}, R C_{j}\right\rangle \in \Gamma$ and $\left.R C_{j} \neq \emptyset\right\}$.

Definition 3.10 Let $\Delta \in\{L L, R L, I L\}, A: x$ be a declarative unit, $T$ be a (possibly empty) set of initial assumptions, $A: x$ is derivable from $T$ in the substructural logic $\Delta$, written $T \vdash_{\Delta} A: x$, if there exists a tuple $\left\langle\Pi,\left\{\left\langle I C_{1}, R C_{1}\right\rangle, \ldots,\left\langle I C_{m}, R C_{m}\right\rangle\right\}\right\rangle$, where $\Pi=\left\{\alpha_{1}, \ldots, \alpha_{n}\right\}$ is a structural derivation of $A: x$ from $T$ with domain $D$ and $\left\{\left\langle I C_{1}, R C_{1}\right\rangle, \ldots,\left\langle I C_{m}, R C_{m}\right\rangle\right\}$ is the set of constraints associated with $\Pi$, such that the set $\left\{\left\langle I C_{1}, R C_{1}\right\rangle, \ldots,\left\langle I C_{m}, R C_{m}\right\rangle\right\}$ is satisfied in the labelling algebra $\mathcal{L}_{\Delta}^{D}$. The

$4_{\text {Extending- }} \mathcal{Y}_{\Delta}^{D}$ with ICs means adding the ICs to the relation $\sqsubseteq$ and closing it under the properties 2 and 3 of Definition 12.11. 
tuple $\left\langle\Pi,\left\{\left\langle I C_{1}, R C_{1}\right\rangle, \ldots,\left\langle I C_{m}, R C_{m}\right\rangle\right\}\right\rangle$ is called a correct derivation of $A: x$ from $T$ in $\Delta$.

Theorems are formulae proved to be derivable with the atomic label 1 from an empty set of initial assumptions. This is formally defined as follows.

Definition 3.11 Let $\Delta \in\{L L, R L, I L\} . A$ formula $A$ is a theorem of $\Delta$ if the declarative unit $A: 1$ is derivable from an empty theory in $\Delta$. This is sometimes written as $\emptyset \vdash_{\Delta} A: 1$, or simply $\vdash_{\Delta} A: 1$.

\subsection{Uniformly Coping with Different Logics (An Example)}

The uniform property of the labelled natural deduction rules, claimed in the previous section, is here illustrated with an example. This consists of taking a formula, known to be a theorem of relevance logic but not of linear logic, constructing a structural derivation, which is the same whatever underlying logic, and describing how different logics are accommodated within the system by means of appropriate solving processes on the label constraints.

The formula under consideration is $A \rightarrow((A \otimes A \rightarrow B) \rightarrow B)$. By Definition 3.11 , to show that this formula is a theorem of LL or of RL, it is necessary to show that there exists a derivation with domain $D$ of the declarative unit $A: 1$ whose set of label constraints is satisfied in $\mathcal{L}_{\mathrm{LL}}^{D}$ or $\mathcal{L}_{\mathrm{RL}}^{D}$.

The case of LL is considered first. A structural derivation is given in Figure whose domain $D=\{a, b, 1\}$, and set of associated constraints is $\left\{\left\langle\emptyset, a \sqsubseteq \gamma_{1}\right\rangle,\langle\emptyset, a \sqsubseteq\right.$ $\left.\left.\gamma_{2}\right\rangle,\left\langle\emptyset, \gamma_{1} \circ \gamma_{2} \sqsubseteq \gamma_{3}\right\rangle,\left\langle\emptyset, b \circ \gamma_{3} \sqsubseteq 1 \circ a \circ b\right\rangle\right\}$. The reader is urged to reconstruct the derivation by working backwards from the initial goal in line 9 . The required constraints extracted from this set are given in (3. $\overline{3}$.

$$
\left\{a \sqsubseteq \gamma_{1}, \quad a \sqsubseteq \gamma_{2}, \gamma_{1} \circ \gamma_{2} \sqsubseteq \gamma_{3}, \quad b \circ \gamma_{3} \sqsubseteq 1 \circ a \circ b\right\}
$$

\begin{tabular}{|c|c|c|}
\hline \multicolumn{3}{|c|}{${ }_{1} A: a$} \\
\hline 2 & $A \otimes A \rightarrow B: b$ & \\
\hline 3 & \begin{tabular}{ll||l}
$A: \gamma_{1}$ & $\sqrt{ } a \sqsubseteq \gamma_{1}$ & $A: \gamma_{2}$ \\
\end{tabular} & $\sqrt{ } a \sqsubseteq \gamma_{2}$ \\
\hline 4 & $A \otimes A: \gamma_{3}$ & $(\otimes \mathcal{I}) \gamma_{1} \circ \gamma_{2} \sqsubseteq \gamma_{3}$ \\
\hline & $A \otimes A: \gamma_{3}$ & (Lemma) \\
\hline & $B: b \circ \gamma_{3}$ & $(\rightarrow \mathcal{E})$ \\
\hline & $B: 1 \circ a \circ b$ & $\sqrt{ } b \circ \gamma_{3} \sqsubseteq 1 \circ a \circ b$ \\
\hline 8 & $(A \otimes A \rightarrow B) \rightarrow B: 1 \circ a$ & $(\rightarrow \mathcal{I})$ \\
\hline 9 & $A \rightarrow((A \otimes A \rightarrow B) \rightarrow B): 1$ & $(\rightarrow \mathcal{I})$ \\
\hline
\end{tabular}

Fig. 1. An Example of structural derivation. 
This derivation is a correct derivation in LL if and only if its associated set of constraints is satisfied in $\mathcal{L}_{\mathrm{LL}}^{D}$. In general, a set of constraints is satisfied if each required constraint $x \sqsubseteq y$ "succeeds" for some instantiation values of the variables occurring in $x$ and in $y$, with respect to the properties of the underlying labelling algebras extended with its associated imposed constraints (if any). These values are called solutions of the constraints and the process of searching for solutions of a given required constraint is called the solving process. The associated labelling algebras of LL include the commutativity property of $\circ$. In solving a required constraint, the two properties of associativity and commutativity need be taken into account. This allows, for example, a required constraint of the form $a \circ b \sqsubseteq \gamma \circ a$ to succeed for $\gamma=b$, as by commutativity it could be reduced to the constraint $b \circ a \sqsubseteq \gamma \circ a$. In the above example derivation, the required constraints $a \sqsubseteq \gamma_{1}$ and $a \sqsubseteq \gamma_{2}$ are satisfied in $\mathcal{L}_{\mathrm{LL}}^{D}$ only if $\gamma_{1}=a$ and $\gamma_{2}=a$ respectively, and the constraint $\gamma_{1} \circ \gamma_{2} \sqsubseteq \gamma_{3}$ is satisfied only if $\gamma_{3}$ is instantiated to $a \circ a$. The last constraint $b \circ \gamma_{3} \sqsubseteq 1 \circ a \circ b$ can be reduced to $b \circ a \circ a \sqsubseteq 1 \circ a \circ b$, which, by the identity property of the element 1 , is equivalent to $b \circ a \circ a \sqsubseteq \bar{a} \circ b$. This constraint is not true in $\mathcal{L}_{\mathrm{LL}}^{D}$. The set of constraints associated with the structural derivation given in Figure ${ }_{11}^{\prime 1}$ is therefore not satisfied in $\mathcal{L}_{\mathrm{LL}}^{D}$ and the proof is not a correct derivation in LL.

To show that the same formula is a theorem of Relevance Logic it is necessary to show that there exists a correct derivation of the declarative unit $A \rightarrow((A \otimes A \rightarrow$ $B) \rightarrow B): 1$ in RL. This means to show that there exists a structural derivation with domain $D$ whose set of associated constraints is satisfied in $\mathcal{L}_{\mathrm{RL}}^{D}$. The same structural derivation, with the same domain $D$, given in Figure ilis is used also in this case - rule applications do not depend on the particular underlying logic. However, to show that it is a correct derivation in RL, it is necessary to show that the set of required constraints in $(3.1)$ is satisfied in $\mathcal{L}_{\mathrm{RL}}^{D}$. This labelling algebra includes both the commutativity and contraction properties of $\circ$. The contraction property allows the left-hand side of a constraint to contain more occurrences than the right-hand side, of any label appearing in the right-hand side. So, for example, a required constraint of the form $a \circ b \circ b \sqsubseteq a \circ b$, which would not succeed in $\mathcal{L}_{\mathrm{LL}}^{D}$, does instead succeed in $\mathcal{L}_{\mathrm{RL}}^{D}$.

To retain the solution process adopted in LL and to allow also for contraction, a way of "evening up" the atomic occurrences in the left-hand and right-hand sides of constraints is needed. This is done via the use of slack variables. For each required constraint $x \sqsubseteq y$, an additional variable, denoted with $\delta$ and called a slack variable, is added to its right-hand side, giving the new constraint $x \sqsubseteq y \circ \delta$. This variable can only be unified with the atomic labels contracted in the left-hand side. If no contraction occurs on the left-hand side of the required constraint then the slack variable is unified with 1. Once the instantiations of all the variables (slack and non) occurring in a constraint are found, which satisfy the equality $x=y \circ \delta$ modulo commutativity, it is then necessary to check that the value of the slack variable corresponds to contracted labels. If this checking fails than the instantiations found are rejected. This is shown in the solution process described below for the constraints in (3.i. $\mathcal{L}_{\mathrm{RL}}^{D}$.

To check whether the set (3.11) of required constraints is satisfied in $\mathcal{L}_{\mathrm{RL}}^{D}$, it is first necessary to add slack variables to their right-hand sides. This gives the following new set $\left\{a \sqsubseteq \gamma_{1} \circ \delta_{1}, a \sqsubseteq \gamma_{2} \circ \delta_{2}, \gamma_{1} \circ \gamma_{2} \sqsubseteq \gamma_{3} \circ \delta_{3}, \quad b \circ \gamma_{3} \sqsubseteq \gamma \circ a \circ b \circ \delta_{4}\right\}$. The first 
two constraints are satisfied by the instantiation $\gamma_{1}=\gamma_{2}=a$ and $\delta_{1}=\delta_{2}=1$. (No contraction takes place here.) The third constraint becomes, under this instantiation, $a \circ a \sqsubseteq \gamma_{3} \circ \delta_{3}$. In this case $\gamma_{3}$ can be either $a$ or $a \circ a$ or 1 . The first instantiation would make $\delta_{3}$ equal to $a$, which means that a contraction of one occurrence of $a$ has occurred in the left-hand side of the constraint. The second instantiation would make $\delta_{3}$ equal to 1 , which means no contraction has occurred. The third instantiation $\left(\gamma_{3}=1\right)$ would instead make $\delta_{3}=a \circ a$, which means that all the occurrences of $a$ in the left-hand side are included in $\delta_{3}$. The last of these instantiations $-\left\{\gamma_{3}=1, \delta_{3}=a \circ a\right\}-$ is however rejected as a slack variable can only "absorb" contracted occurrences. Substituting either of the other two instantiations (i.e. $\left\{\gamma_{3}=a, \delta_{3}=a\right\}$ and $\left\{\gamma_{3}=a \circ a, \delta_{3}=1\right\}$ ) to the fourth inequality, the solution $\gamma=1$ can be obtained, thus showing that $A \rightarrow((A \otimes A \rightarrow B) \rightarrow B): \gamma$ is a theorem of RL.

This technique of using slack variables on the right-hand side of a required constraint is still applicable in Intuitionistic Logic, as contraction is also a property of the labelling algebras associated with this logic. However, for these labelling algebras $\mathcal{L}_{\mathrm{IL}}$, the solving process has also to take into account the monotonicity property. Monotonicity means that constraints which have atomic elements on the right-hand side additional to those on the left-hand side are satisfied. Thus to use the solution process illustrated above, additional slack variables need also to be added to the left-hand side of a required constraint to even up the additional labels occurring in its right-hand side. A full detailed description of the solving process is given in Section 4.2

From the example of constraints solution given above, it is evident that the order in which the constraints of a given set are solved facilitates their solutions. Solving a constraint of the form $\gamma_{1} \circ \gamma_{2} \sqsubseteq \gamma_{3}$ without knowing any particular instantiation value for any of the three occurring variables is in fact much harder. In Section 4.1 it is shown that for any given set of generated required constraints it is always possible to define an ordering (similar to the one implicitly used here) which facilitates the search for solutions.

\subsection{Resource Abduction}

So far it has been illustrated, via an example, how the same structural derivation can be constructed in different logics and how different logics can be accommodated within the same basic solving process for label constraints. An extension, and more general approach, to this proof system is given by the use of a technique called resource abduction. This consists of showing that for a given formula $A$ there exists a derivation of the declarative unit $A: \gamma$ (for an arbitrary variable $\gamma$ ) instead of the declarative unit $A: 1$. In so doing, some of the constraints generated within the derivation would refer to the variable $\gamma$ and their solutions would include an instantiation value for $\gamma$. If $\gamma=1$ is a solution, then the given formula is a theorem of the underlying logic. If this is not the case, then a solution for $\gamma$ provides information of the atomic formulae that could be added to the given formula (as initial assumptions) in order to prove this new formula to be a theorem of the underlying logic. Consider this technique in the above example. The declarative unit to be proven is $A \rightarrow((A \otimes A \rightarrow B) \rightarrow B): \gamma$. A structural derivation identical to that given in Figure each occurrence of 1 in the labels replaced by $\gamma$. The set of associated constraints is 
again reduced to the set of required constraints given in (3.2i).

$$
\left\{a \sqsubseteq \gamma_{1}, a \sqsubseteq \gamma_{2}, \gamma_{1} \circ \gamma_{2} \sqsubseteq \gamma_{3}, \quad b \circ \gamma_{3} \sqsubseteq \gamma \circ a \circ b\right\}
$$

In the case of LL, the only solution of the constraints in (3.2) is $\gamma_{1}=a, \gamma_{2}=a$, $\gamma_{3}=a \circ a$ and $\gamma=a$. Since the value for $\gamma$ is not 1 , the structural derivation in Figure '1'i' is not a derivation in LL, as already concluded above. However, the value $\gamma=a$, together with the fact that $a$ is a solo parameter for $A$ occurring in the structural derivation, indicates that "adding a missing $A$ assumption" to the initial formula will prove the new formula to be a theorem of LL. Adding such a "missing resource" in the form of " $A \rightarrow$ " to the front of the initial formula would give the new formula $A \rightarrow(A \rightarrow((A \otimes A \rightarrow B) \rightarrow B))$. To prove that this is a theorem of LL, a structural derivation is first constructed, as shown in Figure whose set of associated constraints is reduced to the required constraints given in (3.3.

\begin{tabular}{|c|c|c|}
\hline \multicolumn{3}{|c|}{$1 \quad A: a$} \\
\hline \multicolumn{3}{|c|}{$2 A: a$} \\
\hline \multicolumn{3}{|c|}{$3 \quad A \otimes A \rightarrow B: b$} \\
\hline 4 & \begin{tabular}{l||l|}
$\sqrt{ } a \sqsubseteq \gamma_{1}$ & $A: \gamma_{2}$ \\
\end{tabular} & $\sqrt{ } a \sqsubseteq \gamma_{2}$ \\
\hline 5 & $A \otimes A: \gamma_{3}$ & $(\otimes \mathcal{I}) \gamma_{1} \circ \gamma_{2} \sqsubseteq \gamma_{3}$ \\
\hline 6 & $A \otimes A: \gamma_{3}$ & (Lemma) \\
\hline 7 & $B: b \circ \gamma_{3}$ & $(\rightarrow \mathcal{E})$ \\
\hline 8 & $B: \gamma \circ a \circ a \circ b$ & $\sqrt{ } b \circ \gamma_{3} \sqsubseteq \gamma \circ a \circ a \circ b$ \\
\hline 9 & $(A \otimes A \rightarrow B) \rightarrow B: \gamma \circ a \circ a$ & $(\rightarrow \mathcal{I})$ \\
\hline 10 & $A \rightarrow((A \otimes A \rightarrow B) \rightarrow B): \gamma \circ a$ & $(\rightarrow \mathcal{I})$ \\
\hline 11 & $A \rightarrow(A \rightarrow((A \otimes A \rightarrow B) \rightarrow B)): \gamma$ & $(\rightarrow \mathcal{I})$ \\
\hline
\end{tabular}

FIG. 2. Structural derivation with resource abduction.

$$
\left\{a \sqsubseteq \gamma_{1}, a \sqsubseteq \gamma_{2}, \gamma_{1} \circ \gamma_{2} \sqsubseteq \gamma_{3}, \quad b \circ \gamma_{3} \sqsubseteq \gamma \circ a \circ a \circ b\right\}
$$

A solution of (3.3i) in $\mathcal{L}_{\mathrm{LL}}^{D}$ is $\gamma_{1}=\gamma_{2}=a, \gamma_{3}=a \circ a$ and $\gamma=1$. The existence of the value $\gamma=1$ shows that the new formula is a theorem of LL. The use of such a resource abduction technique does not affect derivations which are already correct with respect to a given logic without resource abduction. For instance, using this technique in the case of RL, the constraints in (3.2. $)$, rewritten so to include slack variables, can still be solved in the same way as shown for the set (3.1. and so proving that the given formula is a theorem of RL. No additional resource is in this case necessary as the initial formula is already a theorem of RL.

This is a very nice illustration of the power of the resource abduction technique used in this proof system. Structural derivations that do not result in being derivations of a given logic may be used to "abduce" other theorems of that logic. To the best 
of the authors' knowledge, there is not theorem prover in the literature with such a characteristic.

\section{Solving Constraints}

In this section formal definitions of the algorithmic procedures adopted to solve a set of label constraints are given for each considered substructural logic. As mentioned in the previous section, the case of LL is the simplest one. Only the properties of associativity and commutativity of the operator o need to be taken into account when defining its algorithmic procedure. For the cases of RL and IL, extensions of the LL's algorithmic procedure are defined which accommodate the additional properties of contraction and monotonicity.

Before going into the details of the algorithmic procedures it is important to briefly discuss the issue of defining such algorithms. It has been stated in the previous sections that within this system the same structural derivation with a domain $D$ can be constructed in different substructural logics, as the "decision" process whether the structural derivation is a correct derivation in $\Delta$ is left to the solving process on the label constraints associated with the structural derivation. To fully satisfy such a decision requirement, the solving process should be able to identify when the given formula is a theorem of $\Delta$ or not. Because of the resource abduction technique, this means providing an algorithm which solves (whenever possible) every required constraint associated with the structural derivation and which terminates giving a possible instantiation (i.e. solution) for the variables in the constraints. When the algorithm terminates with a solution that satisfies each required constraint in the $\mathcal{L}_{\Delta}^{D}$ extended with the associated, instantiated imposed constraints, the derivation is a correct derivation in $\Delta$.

Given a structural derivation $\Pi$ of a declarative unit $A: x$ from a set of initial assumptions $T$, with a domain $D$ and set $C$ of associated constraints, the search of solutions for $C$ is made by the algorithm within the domain $D$. By Definition the set $C$ of constraints is satisfied if there exists an instantiation for its variables in $\mathcal{L}_{\Delta}^{D}$. The solving process is able to find such an instantiation (if one exists) as long as it is allowed for the number of steps to be unlimited. The declarative unit $A: x$ is then proved to be derivable in $\Delta$ from $T$. The solving process is composed of two steps, namely instantiation and expansion. The first one instantiates the variables occurring in a $\mathrm{RC}$, whereas the expansion step allows the generation of a new constraint from a given required constraint, using the information given by its associated imposed constraints. These two steps are described in detail in Sections 4 and 4 respectively. Because of the expansion step, the termination property of this process is not always guaranteed. Examples can in fact be constructed in which infinitely many instantiations of the variables occurring in the RCs can be found using their associated ICs and the properties of the underlying labelling algebra, which would still not satisfy all the label constraints. (See example illustrated in Figure $11 \overline{1}_{1}^{\prime}$.) In such cases the solving process would not terminate and no kind of decision (neither positive nor negative) could be reached. The process is therefore semi-decidable. To control the search for solutions an incremental limit on the number of times ICs are used to generate new possible solutions needs to be imposed.

The following theorem captures this discussion. It is justified by the formal defini- 
tions of the algorithmic procedures and of the solving process given throughout the rest of this section.

Theorem 4.1 Let $\Delta \in\{L L, R L, I L\}, E: \gamma$ be a declarative unit and $T$ a set of initial assumptions. Let $\Pi$ be a structural derivation of $E: \gamma$ from $T$ with domain $D$ and set of associated constraints $\left\{\left\langle I C_{1}, R C_{1}\right\rangle, \ldots,\left\langle I C_{n}, R C_{n}\right\rangle\right\}$. If a finite restriction is imposed on the number of expansion steps, then the solving process terminates. If the set $\left\{\left\langle I C_{1}, R C_{1}\right\rangle, \ldots,\left\langle I C_{n}, R C_{n}\right\rangle\right\}$ of constraints is satisfied in $\mathcal{L}_{\Delta}^{D}$, then there exists such a finite restriction on the number of expansion steps for which the solving process terminates giving the solutions. If the set $\left\{\left\langle I C_{1}, R C_{1}\right\rangle, \ldots,\left\langle I C_{n}, R C_{n}\right\rangle\right\}$ is not satisfied in $\mathcal{L}_{\Delta}^{D}$, then either the solving process terminates with no solution or, there is an infinite number of expansion steps and the process does not terminate.

As a consequence of Theorem 4.1, whenever $T=\emptyset$, if the set of constraints is satisfied and the variable $\gamma$ in the declarative unit $E: \gamma$ is instantiated to 1, then the formula $E$ is a theorem of $\Delta$. If $\gamma$ can only be instantiated with values different from 1, then $E$ has not been shown to be a non-theorem of the underlying logic $\Delta$, as there may be a different structural derivation of $E: 1$. On the other hand, the formula of the form $A_{1} \rightarrow\left(A_{2} \rightarrow\left(\ldots A_{k} \rightarrow E\right) \ldots\right)$, where $A_{i}$ is the sub-formula associated with the i-th element in the instantiation of $\gamma$, is a theorem in $\Delta$.

Before the solving process is applied on a set of generated label constraints, the set of required constraints is ordered to facilitate an easier search for solutions. This ordering procedure is defined first in the following section and the definition of the solving process is then given in Section $\overline{4} . \overline{4}$.

\subsection{Requirements for Required Constraints}

This section shows that required constraints generated within a structural derivation can be brought to a simple format, which facilitates their solutions. Firstly, some remarks about notation. In what follows labels of the form $a_{1} \circ a_{2} \circ \ldots \circ a_{n}$ are written more simply as $a_{1} a_{2} \ldots a_{n}$. Since $\circ$ is associative and commutative $a_{1} a_{2} \ldots a_{n}$ can be interpreted as a multi-set composed of the elements $a_{1}, a_{2}, \ldots$, $a_{n}$. Therefore standard operations on multisets can be used on such terms. Specifically, $a_{1} a_{2} \ldots a_{n}-b_{1} b_{2} \ldots b_{m}$, denotes the label obtained by applying the multi-set "subtraction" operation on the two labels $a_{1} a_{2} \ldots a_{n}$ and $b_{1} b_{2} \ldots b_{m}$. Analogously $x \in a_{1} a_{2} \ldots a_{n}$, denotes a single occurrence of $x$ in the multiset $a_{1} a_{2} \ldots a_{n}$. Moreover, throughout the rest of this paper the notation $v_{L}\left(R C_{i}\right)$ and $v_{R}\left(R C_{i}\right)$ is used to refer to the set of variables occurring on the LHS and to the set of variables occurring on the RHS of a given required constraint $R C_{i}$.

As mentioned in the previous sections a required constraint may contain variables in both its left and right-hand sides. Its satisfiability consists in finding instantiations for each of these variables. In this section it is shown that a set of RCs generated in a structural derivation satisfies some specific requirements (see Definition 4.3 ) which facilitate an ordering and thus an easier search of solutions. Specifically, these requirements guarantee that any generated set of RCs can be ordered in such a way that when solved (within this ordering) each constraint becomes of the form

$$
a_{1} \ldots a_{n} \sqsubseteq b_{1} \ldots b_{m} \gamma
$$


where $\gamma$ is the only variable.

Definition 4.2 Let $\left\{R C_{1}, \ldots, R C_{m}\right\}$ be a set of required constraints. A sequence of variables $v_{1}, \ldots, v_{n}$ can be constructed from the $R C s$ if there is an ordering $R C_{1}, \ldots$, $R C_{m}$ of the $R C$ s such that for each $1 \leq i \leq n v_{i} \in v_{R}\left(R C_{i}\right)$ and $v_{i+1} \in v_{L}\left(R C_{i}\right)$. If $v_{n}=v_{j}$ for some $j<n$ then the sequence is circular.

For example, given the constraints $x_{1} v_{2} \sqsubseteq y_{1} v_{1}, v_{4} x_{2} v_{3} \sqsubseteq y_{2} v_{2}, x_{3} v_{1} \sqsubseteq y_{3} v_{3}, x_{4} \sqsubseteq v_{4}$, where $x_{i}$, for each $1 \leq i \leq 4$ and $y_{j}$, for each $1 \leq j \leq 3$, are sequences of non-variable atomic labels and $v_{h}$, for each $1 \leq h \leq 4$, are variables, the following two sequences of variables can be constructed $v_{1}, v_{2}, v_{3}, v_{1}$, and $v_{1}, v_{2}, v_{4}$, of which the first is a circular sequence.

Definition 4.3 Let $\Pi$ be a structural derivation of $A: \gamma$, with a set $C$ of associated constraints, such that $\left\{R C_{1}, \ldots, R C_{m}\right\}$ is the set of required constraints extracted from $C$. Then, the following are requirements on this set of required constraints:

1. For $\left.1 \leq i \leq m, v_{L}\left(R C_{i}\right)\right)$ is a singleton set.

2. The constraint $R C_{j}$ corresponding to the leftmost innermost sub-proof of $\Pi$ satisfies $v_{L}\left(R C_{j}\right)=\emptyset$.

3. For each $R C_{i}$ and each $v_{i} \in v_{L}\left(R C_{i}\right)$ there is exactly one $R C_{j}$, with $j \neq i$, such that $v_{R}\left(R C_{j}\right)=\left\{v_{i}\right\}$. (The equality is justified by requirement 1.)

4. No circular sequence of variables can be formed from the given set of required constraints.

In particular, requirement (4) of the above definition implies that for each generated required constraint $R C, v_{L}(R C) \cap v_{R}(R C)=\emptyset$.

Lemma 4.4 Any set of required constraints, extracted from the set of constraints associated with a structural derivation, satisfies requirements (1)-(4) given in Definition 4.31.

Proof. (Outline) Each requirement is considered in turn and it is briefly explained why it is satisfied by the natural deduction rules.

Requirement 1 . The introduction of new variables into a label arises only by using the $(\otimes \mathcal{I})$ rule or the (Lemma) rule. Moreover, there is only one new variable introduced into the RHS of any label by these rules. Both the (Tick) and the $(\otimes \mathcal{I})$ rules generate required constraints with a RHS that uses labels already introduced in the derivation. Hence, considering also that the label of the initial goal is a single new variable, the RHS of generated RCs have exactly one variable.

Requirement 2. The uppermost leftmost innermost box ${ }^{1}{ }^{\prime} \mid$ always uses declarative units which are either temporary assumptions or derived from the initial assumptions by the $(\rightarrow \mathcal{E})$ or $(\otimes \mathcal{E})$ rules and therefore the generated labels are always ground. In both cases these labels occur on the LHS of the required constraints generated by either a $(\otimes \mathcal{I})$ rule or a (Tick) rule.

Requirement 3. Variables appearing on the LHS of a RC come from the application of either the (Lemma) or the $(\otimes \mathcal{I})$ rule. In both cases, the variables are new and

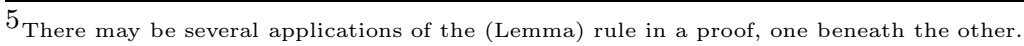


appear only in the sub-derivations of these rules. Within these sub-derivations, such labels would occur on the RHS of a constraint arising from the application of the (Tick) or the $(\otimes \mathcal{I})$ rule. Furthermore, by the argument for Requirement 1 , they can only occur singly. Therefore, there is always exactly one $\mathrm{RC}$ which contains in its RHS the variables used on the LHS of the constraints generated in the sub-derivations.

Requirement 4. Suppose that some circular sequence can be constructed from the RCs generated by $(\otimes \mathcal{I})$ and (Tick) rules. This implies, by Definition 4.2 , that the variables introduced in some of the steps are not new, which is in contradiction with the definition of these rules.

The satisfiability of requirements (1)-(4) argued in the above lemma, allows the set of generated required constraints to be ordered in a list so that the left occurrences of any variable always appear in the list after the right occurrence. This is called an ordered list. This list is formed using the following process:

step 0: Find the set $S_{0}$ of required constraints such that for each $R C_{i} \in S_{0}$, $v_{L}\left(R C_{i}\right)=\emptyset$. (This set is non-empty by requirement (2) of Definition $4.3 i$ ) Let $R_{0}=\bigcup_{R C_{i} \in S_{0}} v_{R}\left(R C_{i}\right)$.

step $k, k \geq 1$ : Find the set $S_{k}$ of required constraints such that for each $R C_{i} \in S_{k}$ $v_{L}\left(R C_{i}\right) \subseteq R_{k-1}$. Let $R_{k}=\left(\bigcup_{R C_{i} \in S_{k}} v_{R}\left(R C_{i}\right)\right) \cup R_{k-1}$.

The above process terminates when for some $k \geq 1$ the generated set $S_{k}$ is empty and all the given required constraints have been considered, so giving the ordered list $S_{0}, S_{1} \ldots, S_{k}$. The termination condition can be shown using the following reasoning by contradiction. Assume that for some $k, k \geq 1$, the associated set $S_{k}$ is empty and that there are still some required constraints not considered in the process. Let $S_{r}$ be the set of these required constraints $\left(S_{r} \neq \emptyset\right)$. Then, by the definition of the above process, this implies that for each $R C_{i} \in S_{r}, v_{L}\left(R C_{i}\right) \nsubseteq R_{k-1}$. Let $R C_{i}$ be one of these constraints, with a variable $\gamma_{1} \in v_{L}\left(R C_{i}\right)$ and $\gamma_{1} \notin R_{k-1}$. By requirement (3) there exists a constraint $R C_{j}$ such that $\gamma_{1} \in v_{R}\left(R C_{j}\right)$. Similarly, $v_{L}\left(R C_{j}\right)$ contains a variable $\gamma_{2}$ such that $\gamma_{2} \notin R_{k-1}$ (else the constraint $R C_{j}$ would belong to $S_{k}$ contradicting the initial assumption). Continuing finding variables in this manner it is possible to form a sequence $\gamma_{1}, \gamma_{2}, \ldots, \gamma_{n}$. This sequence either stops by Definition 4.2 , so yielding a $R C \in S_{k}$, contradicting the initial assumption, or stops because a variable repeats itself. In the second case a contradiction arises with the requirement (4) of Definition 4.3 and Lemma 4.4

To summarise, any set of required constraints generated in a derivation can be ordered in a list, so that each RC will either only have variables on its RHS or, if it has variables on its LHS, these will have occurred on the RHS of some previous $R C$ in the list. The last constraint (i.e. the rightmost in the list) will have as its single RHS variable the label of the initial goal formula. Solving the constraints in the ordering direction "left-to-right" allows then instantiations of variables to be "propagated" throughout the list. The following sections describe how solutions of a given ordered 
list of required constraints can be found, and how the imposed constraints come into play.

\subsection{Instantiating Variables}

In this section, it is assumed that all the sets of imposed constraints are empty. Nonempty sets of imposed constraints are dealt with in Section 'A.3. The propagation of variables' instantiations between required constraints enables variables occurring on the LHS of a required constraint to be instantiated and the constraint itself to be

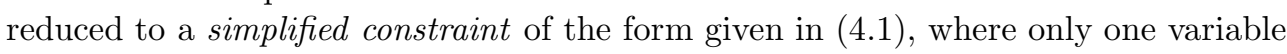
occurs in the RHS. However, in the case of relevance and intuitionistic logics the simplified constraints have also to include auxiliary variables which are introduced in each required constraint either on the RHS or both on the LHS and RHS respectively in order to accommodate the additional properties of contraction and monotonicity. How to instantiate the variables of the resulting simplified constraints is shown below for each of the three logics.

\subsubsection{Linear Logic}

Simplified constraints are of the form $a_{1} \ldots a_{n} \sqsubseteq b_{1} \ldots b_{m} \gamma$. To instantiate the variable $\gamma$ it is sufficient to use the following instantiation algorithm: (i) check first that $b_{1} \ldots b_{m} \subseteq a_{1} \ldots a_{n}$ and (ii) if check (i) succeeds, define $\gamma$ as

$$
\gamma=a_{1} \ldots a_{n}-b_{1} \ldots b_{m}
$$

\subsubsection{Relevance and Intuitionistic Logics}

Slack variables. Simplified constraints include also auxiliary variables, called slack variables. In the case of relevance logic a slack variable is added to the RHS of a simplified constraint in order to cope with contraction. This is illustrated in the following example. Suppose that the RC to solve is abbccc $\sqsubseteq b \gamma$. Without applying contraction and adopting equation $\left(\begin{array}{l}4 . \overline{2} \\ -1\end{array}\right), \gamma$ has to be bound to abccc. But allowing contraction, there are also all the following possibilities: $\gamma=a c, \gamma=a c c, \gamma=a c c c$, $\gamma=a b c, \gamma=a b c c$ and $\gamma=a b c c c$. To find all these solutions, a new variable $\delta_{R}$ is added to the RHS of the constraint. All the labels on the LHS that are not present on the right have to be distributed amongst $\gamma$ and $\delta_{R}$. In the case of intuitionistic logic, slack variables are added to the LHS and to the RHS of each required constraint to accommodate contraction and monotonicity.

In relevance and intuitionistic logics, the instantiation algorithms build upon the one given in linear logic.

Relevance logic. Simplified constraints are of the form $a_{1} \ldots a_{n} \sqsubseteq b_{1} \ldots b_{m} \gamma \delta_{R}$. The instantiation algorithm is analogous to that given in linear logic with the difference that the set difference $a_{1} \ldots a_{n}-b_{1} \ldots b_{m}$ is distributed between $\gamma$ and $\delta_{R}$, with the inclusion restriction that if $x \in \delta_{R}$, then $x \in b_{1} \ldots b_{m} \gamma$. There is always a finite number of solutions.

Using this algorithm in the example given above, for each of the values of $\gamma$ the following respective values of $\delta_{R}$ are found: $b c c, b c, b, c c, c$ and 1 . There are ten more 
candidate solutions, but none of them satisfies the inclusion restriction.

In general, the instantiation of $\delta_{R}$ contains only additional or contracted occurrences of a label - i.e. those that have already appeared elsewhere on the RHS. Notice that unique slack variables are added into each constraint and their values are not propagated as slack variables do not appear on the LHS of any RC.

Intuitionistic logic. Simplified constraints are of the form

$$
\delta_{L} a_{1} \ldots a_{n} \sqsubseteq b_{1} \ldots b_{m} \gamma \delta_{R}
$$

To instantiate the variables $\gamma, \delta_{R}$ and $\delta_{L}$ it is sufficient to use the following instantiation algorithm:

(i) define $\delta_{L}$ as

$$
\delta_{L}=b_{1} \ldots b_{m}-a_{1} \ldots a_{n}
$$

(ii) define $\gamma$ and $\delta_{R}$ as

$$
\gamma \delta_{R}=\delta_{L} a_{1} \ldots a_{n}-b_{1} \ldots b_{m}
$$

distributing the value of $\gamma \delta_{R}$ between $\gamma$ and $\delta_{R}$ with the inclusion restriction that if $x \in \delta_{R}$, then $x \in b_{1} \ldots b_{m} \gamma$.

The instantiation of $\delta_{L}$ facilitates the construction of simplest solutions to equation (4.3i). Consider the following example. Suppose that the constraint to solve is $a b \sqsubseteq$ $\gamma b c$. One solution is $\gamma=a$. Another is $\gamma=a b$ and a more general solution is $\gamma=a \alpha$ (because of monotonicity), where $\alpha$ can be any combination of atomic labels. Now, the solution chosen for $\gamma$ will in general be propagated into the LHS of other RCs. The addition of $\alpha$ will therefore itself be either propagated or contracted until all constraints have been solved. The aim is for the original goal label in the last constraint in the list to be instantiated to 1 , otherwise to the simplest label possible. Restricting the solutions found in IL to be the simplest possible solution means, in this example, that $\alpha$ would be 1 . This is achieved in general by using the definition of $\delta_{L}$ given above.

It is not difficult to check that the above instantiations provide solutions to constraints of the form given in (4.3) in IL, allowing for monotonicity and contraction. This is done by considering the numbers of each atomic label occurring in both sides of the constraints after substitutions are made for $\delta_{L}, \delta_{R}$ and $\gamma$.

\subsection{Using the Imposed Constraints}

In this section, no assumption is made about the set of ICs generated within a structural derivation. This may be empty or non-empty. Imposed constraints are only generated by applications of the $(\otimes \mathcal{E})$ rule to declarative units of the form $A \otimes B: x$. Therefore, they are always of the form $a b \sqsubseteq x$, where $a$ and $b$ are the solo parameters involved in the rule, and where $x$ may or may not contain a variable. The use of an associated imposed constraint ${ }^{16}$ on a given required constraint is called expansion and it is defined as follows.

Definition 4.5 Let $\left(\delta_{L}\right) y \sqsubseteq z$ be a $R C$ and let $a b \sqsubseteq x$ be the associated $I C$ such that $y=a b y_{1}$, where $y_{1}$ is an arbitrary sequence of labels. Then, the $I C$ expands the $R C$

6 Only the ICs paired with a RC can be used in the expansion step on RC. 
into $\left(\delta_{L}\right) x y_{1} \sqsubseteq z$. The $R C$ is sometimes said to be expanded into $\left(\delta_{L}\right) x y_{1} \sqsubseteq z$, and the latter is sometimes called the expanded constraint.

If an expanded constraint $x y_{1} \sqsubseteq z$ is satisfied, then the original required constraint of the form $a b y_{1} \sqsubseteq z$ is also satisfied since $a b y_{1} \sqsubseteq x y_{1} \sqsubseteq z$.

To enforce that the LHS (apart from $\delta_{L}$ if present) of a generated expanded constraint remains ground, only ICs with ground RHSs should be used in an expansion step. As far as this is concerned, ICs of the form $a b \sqsubseteq x$, where $x$ contains a variable, do not need to be used in an expansion step until $x$ is ground. This is justified by the following observation. Any variable $\gamma$ in the RHS of an IC must have been derived from the consequence of some (Lemma) rule application. The associated RC can always be ordered after the RCs generated within the sub-derivation of the (Lemma) rule in which $\gamma$ occurs on the RHS.

It can be seen that the use of ICs increases the possibilities for finding solutions. For example, in LL suppose that, in addition to the RC abbccc $5 \gamma b$, there is the associated IC $b c \sqsubseteq d$. Then, there are two solutions, either $\gamma=a b c c c$ after 0 expansion steps, or $\gamma=a d c c$ after 1 expansion step. Note also that a RC such as $b c a \sqsubseteq \gamma d$ can only be solved by using the IC first, to derive $d a \sqsubseteq \gamma d$.

In RL, contraction might be needed when applying an IC. For example, it is needed in order to solve the following constraints in RL: $a b \sqsubseteq c$ as an imposed constraint and $a b b \sqsubseteq c$ as a required constraint. This is implemented in the same spirit as the instantiation step, by including a slack variable $\delta_{L}$ on the left of the IC. Thus, in RL an imposed constraint $a b \sqsubseteq x$ becomes $a b \delta_{L} \sqsubseteq x$ and expansion matches $a b \delta_{L}$ instead of $a b$, with the restriction $\delta_{L} \subseteq\{a, b\}$.

In IL, $a b \sqsubseteq x$ implies $a \sqsubseteq x$, since $a \sqsubseteq a b$ by monotonicity. Similarly, $b \sqsubseteq x$ is also implied. Hence, in IL an IC of the form $a b \sqsubseteq x$ can be used in an expansion step in three different ways: either to replace an occurrence of $a b$ by $x$ in the RHS of a $\mathrm{RC}$, or to replace an occurrence of $a$ or an occurrence of $b$ by $x$ in the RHS of a RC. This reflects the fact that the $\otimes$ operator in IL behaves exactly as the usual classical conjunction.

\subsection{The Algorithm}

The variable instantiation procedure, described in Section 4.2 together with the expansion process yields the following algorithm, called the solving process, for solving a label constraint problem.

Solving process.

Suppose that $\left\{\left\langle I C_{1}, R C_{1}\right\rangle, \ldots,\left\langle I C_{n}, R C_{n}\right\rangle\right\}$ is the constraint problem to be solved, in which the RCs are ordered as described in Section 4.1 In order to solve the RCs, using the associated ICs, the following two steps are made:

(i) instantiation. Variables in a required constraint are instantiated as described in Section $4.2 ;$

(ii) expansion. An IC is applied to the LHS of a RC (employing the contraction and monotonicity properties as appropriate).

It may be possible to make an arbitrarily large number of expansion steps to a particular required constraint (see for example the problem illustrated in Figure $1 i_{1}^{1} \overline{1}_{1}^{1}$ ). 
Therefore, in order for the solving process to terminate, some kind of limit must be placed on the number of expansion steps made. To better approximate all solutions an incremental limit should be used. Further investigations of a possible upper limit are currently being undertaken.

\section{Correctness of the Natural Deduction Rules}

In this section the set of labelled natural deduction rules, defined in Section shown to be sound with respect to the LKE tableaux system described in [i6]. This is proved by firstly extending the LKE system with an additional rule, called (Tch), secondly showing that this extension is equivalent to the original LKE system and then proving that for each natural deduction derivation there exists a corresponding extended LKE refutation. Before going into the details of the proof a brief description of the LKE rules is given.

\subsection{The LKE System}

The LKE system described in i is a uniform labelled semantic tableaux system for substructural logics which generalises the classical logic KE-tableau system [i] using the LDS approach. Within this system, the refutation rules are common to any substructural logics - they perform the role of operational rules. The standard structural rules of substructural logics are expressed in terms of conditions on the labels, so different logics are captured by just considering different labelling algebras. Note that in [i. i a labelling algebra is defined in terms of a complete lattice. However, the correspondence between theorems in LKE, with respect to a class of labelling algebras associated with a logic $\Delta$, and theorems in the logic $\Delta$ shows that, in the multiplicative fragment, only the labels constructed from the atomic labels appearing in an LKE tree, and the o operator, are necessary. The rule for closing branches takes into account these conditions allowing then some formulae instead of others to be proved. Within a LKE system, a formula $A$ is proved to be a theorem of a given substructural logic if it is possible to show that there exists a refutation of the assumption " $A$ being false at the identity element 1" for the class of labelling algebras associated with the given logic. Such a refutation is a LKE tree starting with the labelled signed formula $F A: 1$, and having all branches closed by applications of the (Cl) rule. In this system there is no clear distinction between semantics and syntax. Semantic notions of a labelling algebra and its consequences, such as the existence of characteristic labels, are integrated into the proof system.

The set of refutation rules, given in $[6]$ and here restricted to the fragment $\{\rightarrow, \otimes\}$ of substructural logics, is listed in Table $\overline{3}$. The rules for the operators $\rightarrow$ and $\otimes$ can be proved $[6,2]$ to be respectively equivalent to the conditions (3) and (4) of Definition 2.2 in Section 2 In the case of $(F \rightarrow)$, the use of the $A$-characteristic atomic label $a$ is justified by the following reasoning. If $x$ does not verify $A \rightarrow B$ then by condition (3) of Definition 2.2 there exists some label which verifies $A$ and which, composed with $x$, does not verify $B$. This implies by the same algebraic property? given in condition (2) of Definition 는. 2 , that there exists a least label, the

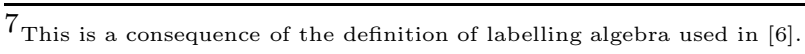




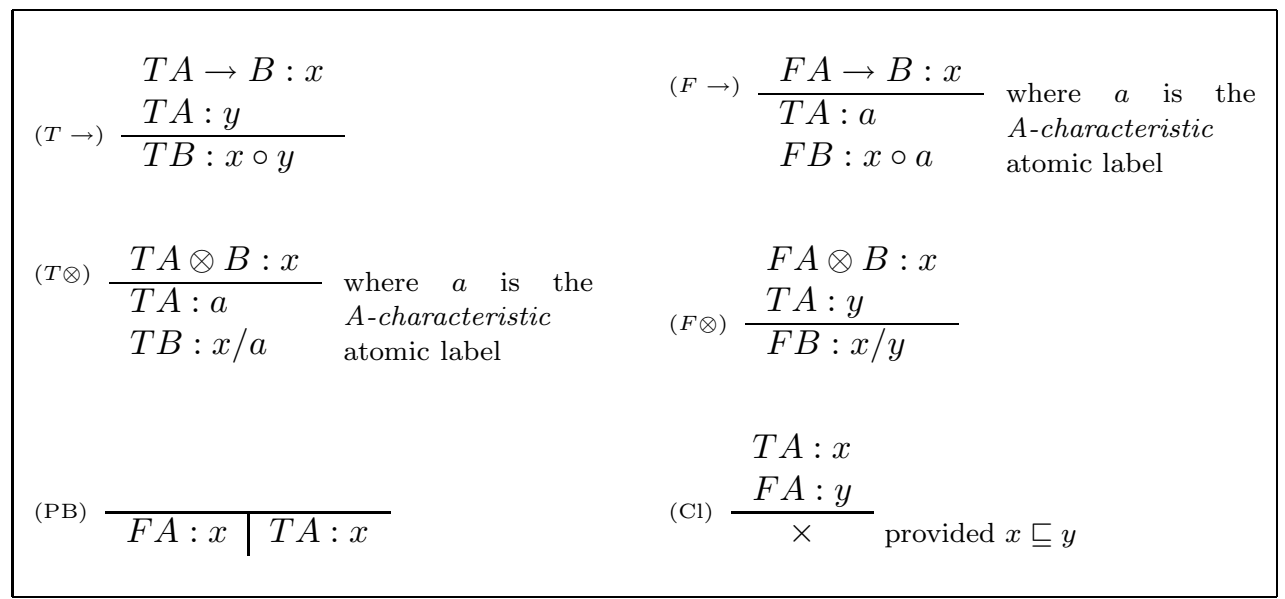

TABLE 3. The LKE rules for the substructural fragment $\{\rightarrow, \otimes\}$.

$A$-characteristic, denoted with $a$, which verifies $A$. The same argument holds for the $(T \otimes)$ rule, but with respect to condition (4) of Definition 2.2 . Notice that in the rules for the multiplicative conjunction $\otimes$, the operator "/", called the residual operator is used. In LKE, the labelling algebra is extended with the characteristic property of / given in $(5,1)$.

$$
y \circ z \sqsubseteq x \text { iff } z \sqsubseteq x / y
$$

Using this operator, instead of deriving, from $T A \otimes B: x, T A: a$ and $T B: b$ with the imposed constraint $a \circ b \sqsubseteq x$, the declarative units $T A: a$ and $T B: x / a$ are derived (or equivalently $T B: b$ and $T A: x / b$ ), without the generation of the IC. The use of the / operator in the natural deduction system has been omitted, as it would have made the whole solving process more complex. This has indeed allowed the development of algorithms for solving required constraints, which is one of the main benefits of the natural deduction system developed in this paper. The (PB) rule expresses the labelled version of the semantic Principle of Bivalence which states that any formula $A$ can be either true or false at each element of the labelling algebra. Application of the (PB) rule can be restricted to sub-formulae of the formulae appearing in the tree. The version used here employs free variables, as described in $\left|\mathbf{6}_{1}\right|$. The $(\mathrm{Cl})$ rule instead states when a branch can be closed, and it is justified by condition (1) of Definition $2.2 i$ given in Section 2 condition $x \sqsubseteq y$, which can be proved to hold using the properties of $\circ$ given by the underlying class of labelling algebras. However, the work in [i] does not cover the issue of finding algorithms for solving these kind of conditions. One example was given in [īi] but only for the case of Linear Logic.

Extending LKE. The LKE system is here extended by adding an extra rule, called (Tch), to those given in Table

$$
\text { (Tch) } \frac{T A: x}{T A: a} \begin{aligned}
& \text { where } a \text { is isacteristic } \\
& \text { atomic label }
\end{aligned}
$$


This rule reflects the algebraic property given in condition (2) of Definition $\overline{2} \overline{2}$, for which $a \sqsubseteq x$, and provides the link between the use of the / operator in the LKE system and the use of the imposed constraints in the natural deduction system. Specifically, in the LKE system the introduction of a label using / by the $(\mathrm{T} \otimes)$ rule corresponds to the introduction of an IC in the natural deduction system. Solo parameters of $L_{L}$ used in a natural deduction derivation are mapped into the LKE characteristic atomic label.

The extended LKE system is equivalent to the original LKE system. This is illustrated in Figure $\overline{3}_{1}^{1}$ and explained below. Arrows 4 and 5 in Figure

$(2)$

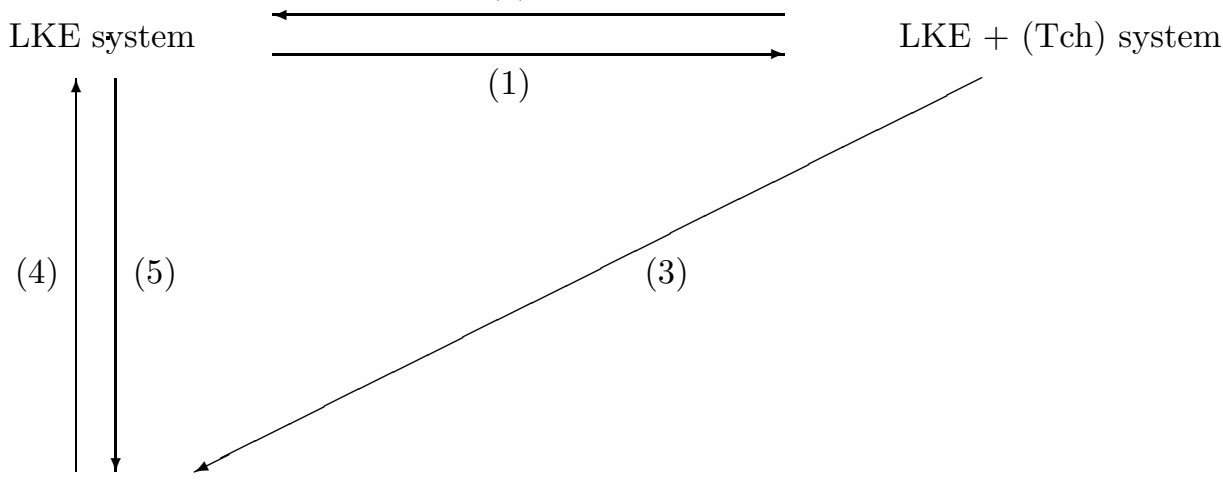

SC system

FIG. 3: Equivalence between the Extended LKE and the original LKE tableaux system.

completeness and soundness property of the LKE system with respect to the sequent calculus, proved in $[6]$ by Propositions 4 and 5 respectively. Arrow 1 shows that for any refutation in the original LKE system there is a refutation in the extended LKE system. This is trivially true. Hence to show the equivalence between the two LKE systems (original and extended) it is just sufficient to show that the extended LKE system is sound with respect to the sequent calculus (arrow 3). This is proved by extending Proposition 5 in [i, the the case of the (Tch) rule. The proof of Proposition 5 in [6] is based on a canonical interpretation, which interprets labels as sets of formulae closed under the sequent calculus derivability relation and declarative units of the form $T A: x$ as $A \in x$, and on a canonical valuation $v$ defined as $v(A, x)=T$ iff $A \in x$. Under this canonical interpretation $T A: x$ holds iff $v(A, x)=T$. Extending Proposition 5 to cover the (Tch) rule requires to show that if $T A: x$ holds then $T A: a$ holds, where $a$ is the $A$-characteristic element in the labelling algebra. Using 
the above canonical interpretation $T A: x$ holds implies $v(A, x)=T$, which, by clause (2) of Definition 2 implies that $v(A, a)=T$ and hence $T A: a$ holds. The reader is referred to [i़in] for further details.

Completeness with respect to LKE. In ini] it has been shown that this system of natural deduction rules is complete with respect to the LKE system. That is, there exists an algorithm which turns a LKE refutation of a theorem $A: 1$, formed in a particular way, into a labelled natural deduction derivation of $A: 1$, showing also that tableau refutation rules can be read as backward reasoning in this labelled natural deduction system. For more details the reader is referred to [2].

\subsection{Soundness with Respect to LKE}

The soundness of the ND system with respect to the extended LKE is shown in this section. The equivalence between the LKE system and the extended LKE system implies the soundness of the ND rules with respect to the LKE system. In the rest of this section "LKE system" will refer to the extended set of LKE rules.

In the following proof, notions of "length of a ND rule" and "length of a derivation" are used. These are defined as follows. The ND rules which do not have sub-derivations in their antecedents, with the exception of the $(\otimes \mathcal{E})$ rule, have length equal to 1 (i.e. these are the (Tick) rule and the $(\rightarrow \mathcal{E})$ rule), the $(\otimes \mathcal{E})$ rule has length equal to 2, and the rest of the ND rules have length equal to the (sum of the) length(s) of the smallest sub-derivation(s) in their antecedent incremented by 1 . For example, the $(\rightarrow \mathcal{I})$ rule has length equal to $1+l_{1}$ where $l_{1}$ is the length of the smallest subderivation in its antecedent, whereas the length of the $(\otimes \mathcal{I})$ rule is equal to $1+l_{1}+l_{2}$ where $l_{1}$ and $l_{2}$ are respectively the lengths of the smallest left-hand side and righthand side sub-derivations in the rule's antecedent. The length of a given arbitrary derivation is thus equal to the sum of the lengths of the inference rules used in that derivation. Notice that, as in the standard natural deduction, a structural derivation with length equal to 0 is a derivation with no inference rule application, with an empty set of constraints and with the goal being a declarative unit already belonging to the given set of initial assumptions.

The proof of Theorem 5 uses a mapping between ND derivations and LKE trees defined as follows. Each label $f$ of assumptions of the form $F: f$, which occurs in a ND derivation, is mapped to a corresponding label $f$ in a signed formula $T F: f$, where $f$ is now the $F$-characteristic label. For any other non atomic label the mapping is extended in an obvious way. The restriction imposed on the solo parameters in the ND rules guarantees this mapping to be a one-to-one function. The proof is in the same spirit as that used in for classical logic.

Theorem 5.1 Let $\Delta$ be a given substructural logic, let $T$ be a set of initial assumptions of the form $\left\{A_{1}: a_{1}, \ldots, A_{n}: a_{n}\right\}$ and let $B: z$ be a declarative unit. If $T \vdash_{\Delta} B: z$ then there exists a closed LKE tableau refutation for $T A_{1}: a_{1}, \ldots, T A_{n}: a_{n}, F B: z$.

Proof. Let $\left\langle\left\{\alpha_{1}, \ldots, \alpha_{k}\right\},\left\{\left\langle I C_{1}, R C_{1}\right\rangle, \ldots,\left\langle I C_{n}, R C_{n}\right\rangle\right\}\right\rangle$, with $k \geq 1$ and $n \geq 0$, be the smallest derivation of $B: z$ in the logic $\Delta$ with length $l$. The proof is by induction on $l$.

\section{Base Case}

The base case is when $l=0$, i.e. there is no inference rule application. Therefore 
for some $1 \leq i \leq n, B: z=A_{i}: a_{i}$ (i.e. $B=A_{i}$ and $z=a_{i}$ ). Hence, there is also a closed LKE tableau refutation for the set $T A_{1}: a_{1}, \ldots, T A_{n}: a_{n}, F B: z$, given by one application of the $(\mathrm{Cl})$ rule between $A_{i}: a_{i}$ and $B: z$.

\section{Inductive Step}

Suppose that $l>0$ and that the theorem holds for any smallest derivation of length less than $l$. The proof is then by cases for any rule application on the last step, which generates $\alpha_{k}$. (For simplicity, in each of these cases the initial theory $T$ is subsumed in both the natural deduction derivations and the corresponding LKE trees.)

Case 1: (Tick) rule.

There exists a declarative unit of the form $B: x$, for some label $x$, with its associated constraints, which is part of the derivation $\left\{\alpha_{1}, \ldots, \alpha_{k-1}\right\} . \alpha_{k}$ is instead given by the declarative unit $B: z$ and associated constraints $\left\langle\Gamma_{B: z}, I C_{B: z}\right\rangle$, such that $R C_{B: z}=$ $\{x \sqsubseteq z\}$. A LKE refutation of $F B: z$ can be constructed starting with $F B: z$ and the set of initial assumptions $T$. A (PB) rule is applied on $B: x$. The left branch closes by the inductive hypothesis using the part of the natural deduction derivation, denoted by $\Pi_{1}$, which has inferred $B: x$. The right branch closes with the application of a $(\mathrm{Cl})$ rule. The condition of the $(\mathrm{Cl})$ rule holds for the following reason. The rule applications, before $B: z$, that generate in the natural deduction derivation the ICs used to solve $x \sqsubseteq z$ can be reconstructed, by inductive hypothesis, in the right branch of the LKE tree. These generated ICs correspond to properties of the labelling algebra involving the residual operator, which can be equally used to solve the constraint $x \sqsubseteq z$.
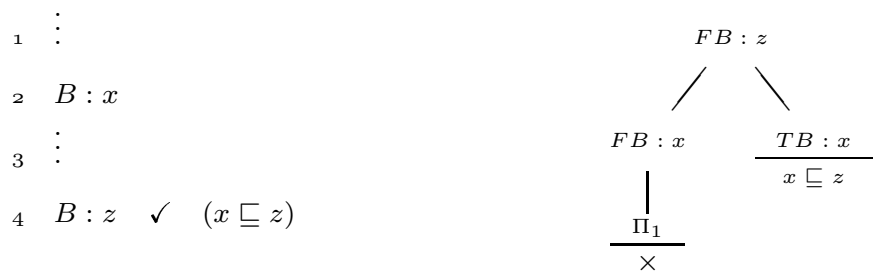

FIG. 4. Correspondence between labelled ND and LKE for the (Tick) rule.

Case 2: $(\rightarrow \mathcal{E})$ rule.

In this case, the constrained declarative unit $\alpha_{k}$ is composed of a declarative unit of the form $B: x \circ y$ and associated constraints. Two constrained declarative units, respectively composed of a declarative unit of the form $D \rightarrow B: x$ and associated constraints and a declarative unit of the form $D: y$ and associated constraints, are part of the derivation $\left\{\alpha_{1}, \ldots, \alpha_{k-1}\right\}$. A LKE refutation of $F B: x \circ y$ can be constructed, which starts with $F B: z$ and the set of initial assumption $T$. A (PB) rule is applied on $D \rightarrow B: x$. The left branch closes by the inductive hypothesis using the part of the natural deduction derivation, denoted with $\Pi_{1}$, which has inferred $D \rightarrow B: x$ and whose length is less than $l$. On the right branch another (PB) rule is applied on $D: y$. Its left branch closes by inductive hypothesis using the part of the natural deduction derivation, denoted with $\Pi_{2}$, which has inferred $D: y$, whereas its right branch closes with the application of $(T \rightarrow)$ and $(\mathrm{Cl})$ rules as shown in Figure 

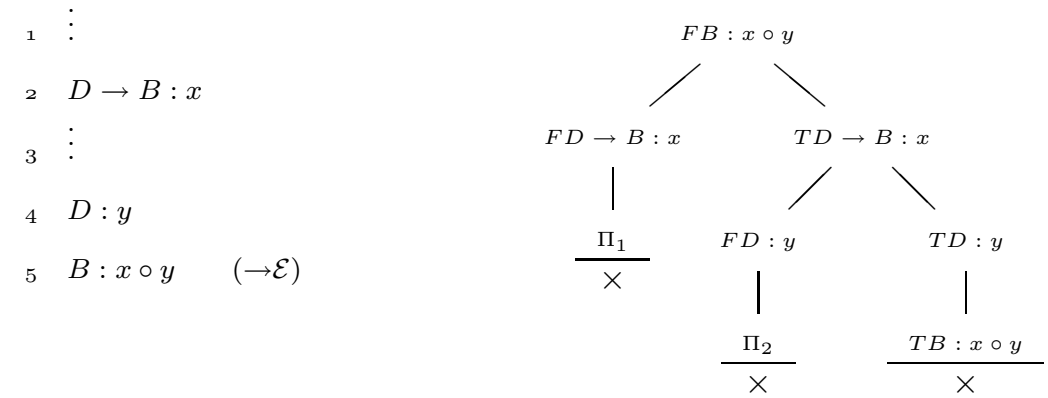

FIG. 5. Correspondence between labelled ND and LKE for the $(\rightarrow \mathcal{E})$ rule.

Case 3: $(\otimes \mathcal{E})$ rule.

There exists a declarative unit of the form $D \otimes B: x$, for some label $x$, with its associated constraints, which is part of the derivation $\left\{\alpha_{1}, \ldots, \alpha_{k-1}\right\} . \alpha_{k}$ is instead a constrained declarative unit composed of either the declarative unit $D: d$ and associated constraints or the declarative unit $B: b$ and associated constraints, where $d$ and $b$ are solo parameters. In either case the new imposed constraint $d \circ b \sqsubseteq x$ belongs to their respective constraints. Consider the first case where $d \circ b \sqsubseteq x \in I C_{D: d}$. A LKE refutation of $F D: d$ can be constructed by starting with $F D: d$ and applying a (PB) rule on $D \otimes B: x$. The left branch closes by inductive hypothesis using the part of the natural deduction derivation, denoted with $\Pi_{1}$, which has inferred $D \otimes B: x$. The right branch closes after the application of a $(T \otimes)$ rule. This is shown in Figure $\overline{\underline{b}}_{r}^{1}$ The second case, when $\alpha_{k}$ is composed of the declarative unit $B: b$, can be proved using an analogous argument, with the addition of a (Tch) rule application in the right branch from $T B: x / d$, which derives $T B: b$. The underlying labelling algebra will satisfy in this case the property $b \sqsubseteq x / d$, which is equivalent to $b \circ d \sqsubseteq x$.

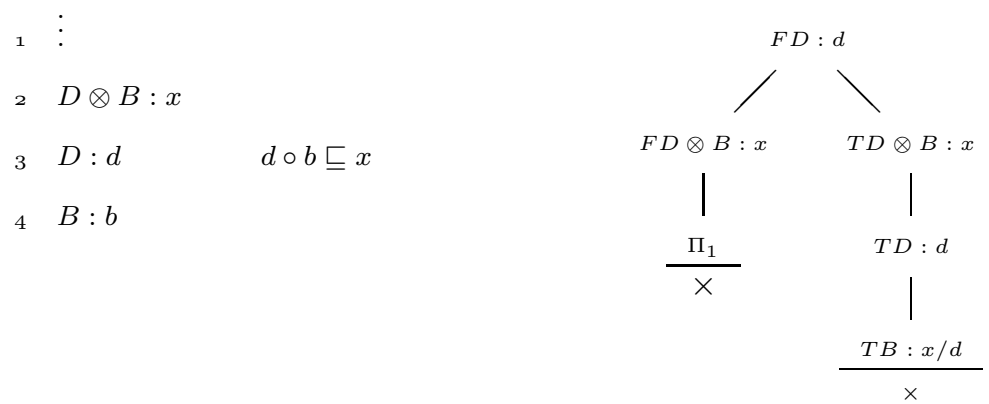

FIG. 6. Correspondence between labelled ND and LKE for the $(\otimes \mathcal{E})$ rule.

Case 4: $(\rightarrow \mathcal{I})$ rule.

In this case, there exists a sub-derivation of $C: z \circ b$, with associated constraints, from $T \cup\{B: b\}$. The constrained declarative unit $\alpha_{k}$ is composed of a declarative unit 
of the form $B \rightarrow C: z$ with associated constraints. A LKE refutation of $F B \rightarrow C: z$ can be constructed by starting with $F B \rightarrow C$ : $z$, adding the assumption $T B$ : $b$, applying the $(F \rightarrow)$ rule and then completing the tree using the sub-derivation, denoted with $\Pi_{1}$, that has proved $T C: z \circ b$. This is shown in Figure $\overline{7}_{1}$. The branch closes by inductive hypothesis on $\Pi_{1}$, since this sub-derivation has length strictly less than $l$.
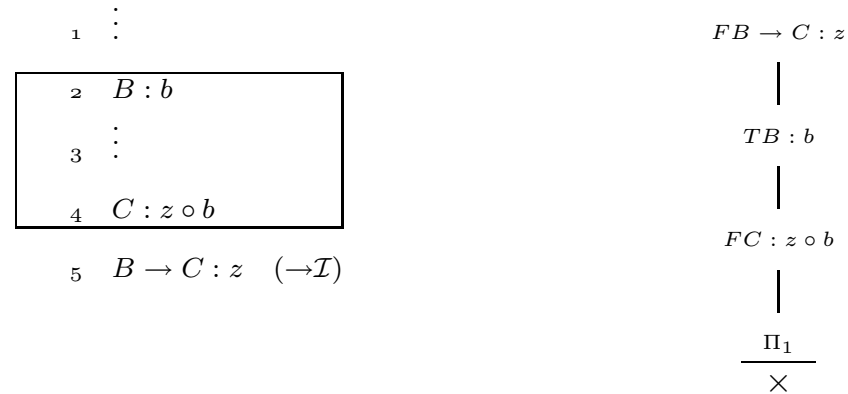

FIG. 7. Correspondence between labelled ND and LKE for the $(\rightarrow \mathcal{I})$ rule.

Case 5: $(\otimes \mathcal{I})$ rule.

In this case, there exists a sub-derivation from $T$ of $B: \gamma_{1}$, with associated constraints, and of $C: \gamma_{2}$ with its associated constraints. The constrained declarative unit $\alpha_{k}$ is composed of a declarative unit of the form $B \otimes C: z$ with associated constraints, such that $R C_{B \otimes C: z}=\left\{\gamma_{1} \circ \gamma_{2} \sqsubseteq z\right\}$. A LKE refutation of $F B \otimes C: z$ can be constructed in the way described below. Start with $F B \otimes C: z$ and apply a (PB) rule on $B: \gamma_{1}$. The left branch closes by inductive hypothesis on the part of the ND derivation that proves $B: \gamma_{1}$ (denoted with $\left.\Pi_{1}\right)$. In the right branch a $(F \otimes)$ rule is applied deriving $F C: z / \gamma_{1}$. Then a $(\mathrm{PB})$ rule is applied on $C: \gamma_{2}$, of which the left branch closes by inductive hypothesis on the part of the ND derivation that proves $C: \gamma_{2}$ (denoted with $\Pi_{2}$ ), and the rightmost branch closes for the following reason. The natural deduction rules that generate the set of imposed constraints $I C_{B \otimes C: z}$ used to resolve the required constraint $\gamma_{1} \circ \gamma_{2} \sqsubseteq z$, can be reconstructed in this branch. This set of imposed constraints corresponds to properties of the labelling algebra involving the residual operator, which allow the closure constraint $\gamma_{2} \sqsubseteq z / \gamma_{1}$ (equivalent to $\gamma_{1} \circ \gamma_{2} \sqsubseteq z$ ) to be solved.

Case 6: (Lemma) rule.

The constrained declarative unit $\alpha_{k}$ is composed of a declarative unit of the form $B: \gamma$ with associated constraints. In this case there exists a sub-derivation of $B: \gamma$ from $T$ with length strictly less then $l$. A LKE refutation of $F B: \gamma$ can be constructed by starting with $F B: \gamma$ and making an application of $(\mathrm{PB})$ on $B: \gamma$. The left branch closes by the inductive hypothesis using the natural deduction derivation, denoted by $\Pi_{1}$, that proves $B: \gamma$, whereas the right branch closes immediately. This is shown in Figure $\overline{9}$.

As an example of the correspondence between natural deduction and the extended 

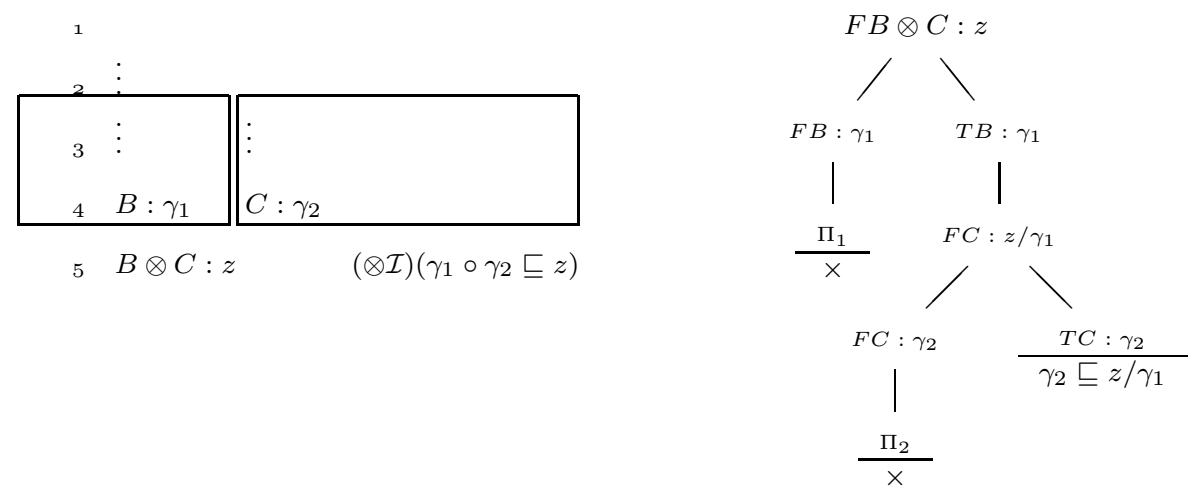

FIG. 8. Correspondence between labelled ND and LKE for the $(\otimes \mathcal{I})$ rule.
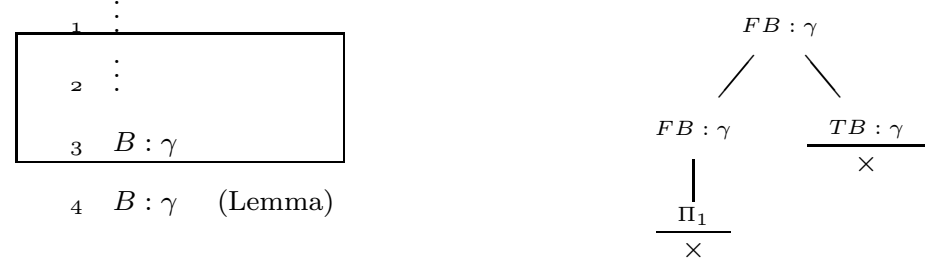

FIG. 9. Correspondence between labelled ND and LKE for the (Lemma) rule.

LKE system, Figure $1 \overline{1} \overline{0}_{1}^{\prime}$ shows a tableau corresponding to the natural deduction derivation given in Figure ${ }_{11}^{1}$, but with $\gamma$ replacing each occurrence of 1 in the labels. In this LKE refutation the rightmost branch closes using the required constraint $b \circ \gamma_{3} \sqsubseteq \gamma \circ a \circ b$. The leftmost branch, under the node $F A \otimes A: \gamma_{3}$, closes using the required constraint $a \sqsubseteq \gamma_{1}$, the left branch under the node $F A: \gamma_{3} / \gamma_{1}$, closes using the required constraint $a \sqsubseteq \gamma_{2}$, whereas the right branch, under the node $F A: \gamma_{3} / \gamma_{1}$, closes using the required constraint $\gamma_{2} \sqsubseteq \gamma_{3} / \gamma_{1}$.

\section{Conclusion}

This paper has shown how a uniform proof method for substructural logics based on natural deduction can be defined using the LDS approach. This system is sound and complete with respect to the LKE system. These properties, together with the correspondence of the LKE system with respect to the sequent calculus, proved in [6] sequent calculus for the class of substructural logics described in [i]

However, the two proof theoretic approaches (ND and LKE system) present significant differences. This is briefly discussed in the following section where an example illustrating such differences is also given. 
6.

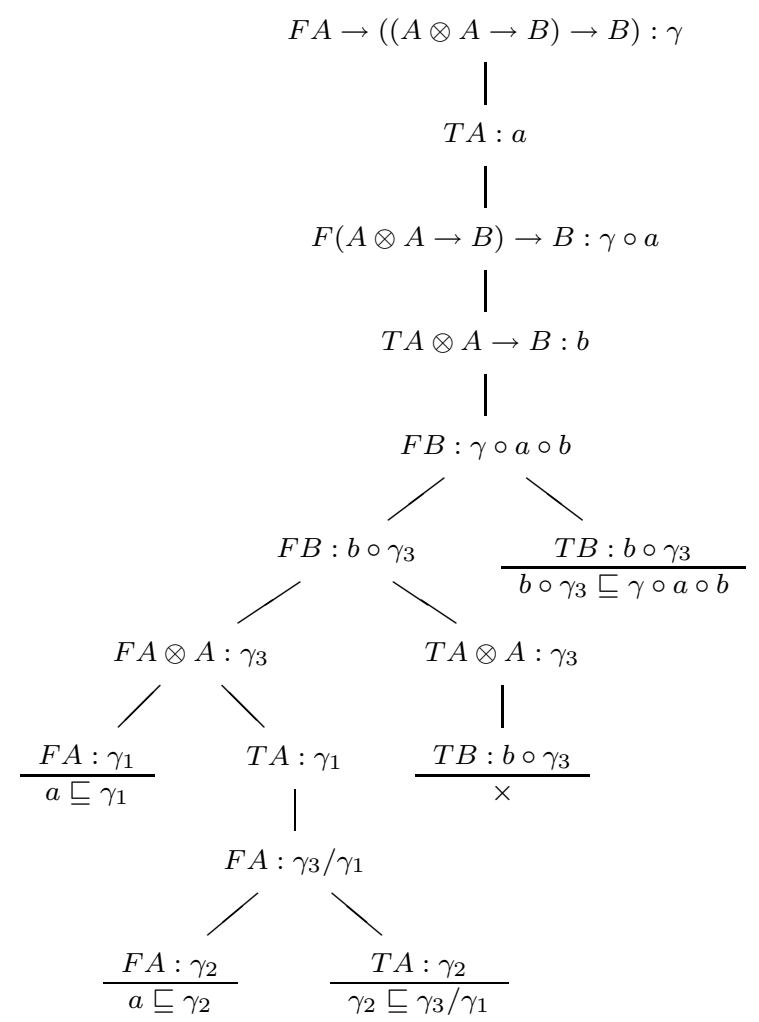

FIG. 10. LKE tree corresponding to the ND derivation of Figure

\subsection{Comparison between ND and LKE Systems}

One of the features of the natural deduction approach described in this paper is that for a given derivation many different solutions for the variable label of the initial goal can be found, which satisfy the associated set of label constraints. This is due to the fact that in the solving process no limit is fixed "a priori" to the number of expansion steps which can be applied to a given set of required constraints. Often, additional applications of the expansion step lead to additional solutions, for the same associated structural derivation. In the LKE system, no explicit use is made of the imposed constraints. The expansion step of the ND's solving process, which uses a generated IC, corresponds instead to additional applications of LKE refutation rules. Consequently, a single ND structural derivation can correspond to more than one (and possibly to an infinite number of ) closed standard LKE trees'. This is shown in the following example.

Consider the ND derivation, given in Figure $11 \overline{1}$, of the declarative unit $E: \gamma$ from the set of initial assumptions $\{A: a, \quad A \rightarrow D: \bar{d}, \quad D \rightarrow E: e, \quad(A \rightarrow D) \rightarrow(D \rightarrow$ $E) \rightarrow(A \rightarrow D) \otimes(D \rightarrow E): c\}$. There is just one required constraint of the form

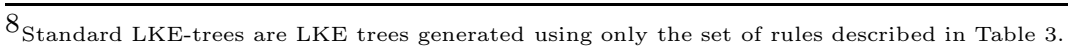


$e \circ d \circ a \sqsubseteq \gamma$ with the associated imposed constraint $d \circ e \sqsubseteq c \circ d \circ e$. In the case of LL,

$1 \quad A: a$

2 $A \rightarrow D: d$

$3 \quad D \rightarrow E: e$

$4 \quad(A \rightarrow D) \rightarrow((D \rightarrow E)$

$\rightarrow(A \rightarrow D) \otimes(D \rightarrow E)): c$

$5 \quad(D \rightarrow E) \rightarrow$

$(A \rightarrow D) \otimes(D \rightarrow E): c d$

$6 \quad(A \rightarrow D) \otimes(D \rightarrow E): c d e$

$7 \quad D: d a$

$8 \quad$ E : eda

$9 \quad E: \gamma$

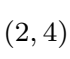

$\left(\begin{array}{ll}31, \\ 3\end{array}\right.$

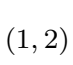

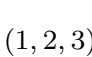

$\checkmark$ eda $\sqsubseteq \gamma$

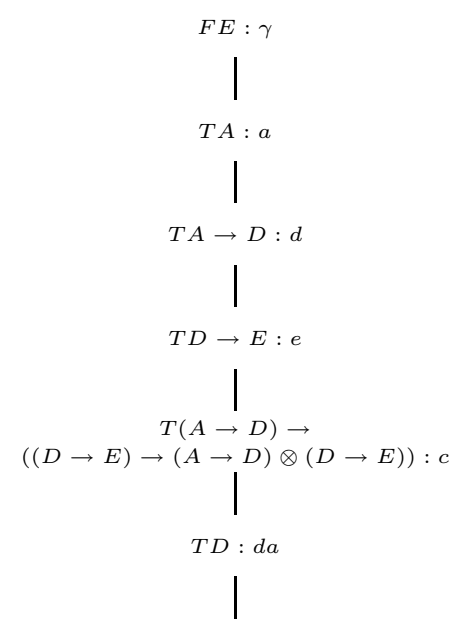

(*) $\mathrm{IC}=d e \sqsubseteq c d e$ from (6), (2) and (3)

$A \rightarrow D: d$ and $D \rightarrow E: e$

are already present by step (6)

and so are not added again.
TE : eda (i)

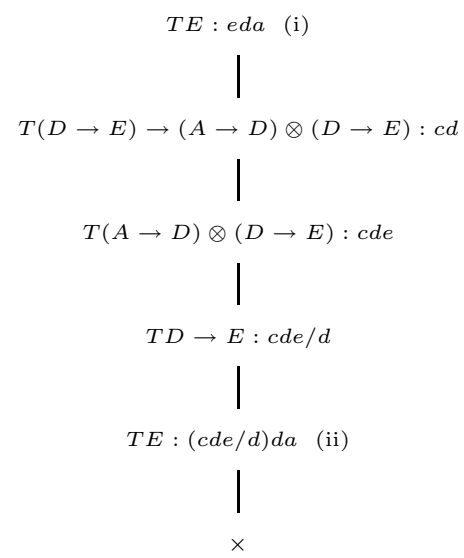

FIG. 11. Example of structural derivation and standard LKE-tree(s)

there are many solutions for the variable $\gamma$ which satisfy the label constraints, namely $\gamma=e d a, \gamma=e c d a, \gamma=e c c d a$ and so on. (For simplicity the o operator has been omitted.) Each of these solutions is obtained by making use of the imposed constraint $d e \sqsubseteq c d e$ none, one or more times. (In RL and IL all solutions which have at least one occurrence of $c$ are equivalent because of the contraction property.) Notice that if in this example the variable $\gamma$ had been the particular label adeccf, for some arbitrary initial assumption $F: f$, then no solution could have been found; yet the imposed constraint $d e \sqsubseteq c d e$ could have been applied infinitely many times to the label eda, but never yielding adecc $f$. This shows once more the semi-decidability of the solving process algorithm.

When a comparison is made with the standard LKE system, it is seen that a single natural deduction structural derivation, such as the one on the left in Figure i1 i i', can correspond to more than one (and possibly to an infinite number of) closed "standard" 
LKE-trees. Examples of two LKE refutations are given, within one tree in the righthand diagram of Figure $\overline{1}_{1} \overline{1}_{r}^{\prime}$ These are obtained by terminating the tree at (i) and (ii) respectively. In this example, supposing that the underlying logic is LL, each solution of the variable label $\gamma$ corresponds to a different standard LKE-tree, depending on the number of times the signed labelled formula $T(D \rightarrow E) \rightarrow(A \rightarrow D) \otimes(D \rightarrow E): c d$ (shortened to $T X$ ) is used. The first solution, $\gamma=e d a$, corresponds to a tree in which such a signed labelled formula $T X$ is used no times (i.e. in this case closure is made at the step (i)); on the other hand the second solution, $\gamma=e c d a$, corresponds to a tree in which the signed labelled formula $T X$ is used once and the derived signed labelled formula $D \rightarrow E: c d e / d$ is used to derive $E:(c d e / d) d a$ (in this case closure is made at step (ii)). Further solutions correspond to bigger treest.

This correspondence between the ND system and the LKE system implies that if for a given structural derivation the solving process does not terminate (e.g. there is no solution to a given set of label constraints) the set of LKE trees reflecting the natural deduction structural derivation would also not terminate.

Additional observations about the two systems are: (i) use of free variables is made in both systems (i.e. in the ND (Lemma) rule and in the LKE (PB) rule) and (ii) limitations, as to the number of times rules are used in a proof, are still to be thoroughly investigated in both systems. As far as (i) is concerned, in [i, it has been observed that the use of free variables in the $(\mathrm{PB})$ rule can vastly improve the practicality of the method. That is, instead of "guessing" the value of the label $x$ to use in a $(\mathrm{PB})$ rule application, a free variable $\gamma$ can be used; $\gamma$ is treated within the proof as a ground label, and it is only at the closure step that a suitable value for it is given using the closure inequation. The soundness and completeness of LKE, with respect to the sequent calculus, shows that only values for $\gamma$ involving $\circ$ and / are necessary. This corresponds to the use of a free variable in the ND (Lemma) rule. However in the ND system this approach is taken even further, allowing free variables to be used also in the $(\otimes \mathcal{I})$ rule. Simple label inequations involving only the $\circ$ operator generated by the closure rule can be solved in the LKE system using algorithms based on the AC-unification technique [1] For more complex inequations involving the / operator no algorithm has, to the authors' knowledge, yet been reported. In the ND approach, the solving process is much simpler, because natural deduction derivations are more structured. This structural feature facilitates the definition of an ordering procedure on the generated label constraints which simplifies the instantiation process and therefore the search for solutions. A similar analysis of "structured LKE trees", but for the $\rightarrow$ operator only, is shown in [i]1].

However, two important practical difficulties still remain when incorporating free variables into the LKE method. The first one is how to limit the applications of the rules and still retain completeness. (For example, $(\mathrm{T} \rightarrow)$ may be applied indefinitely to $A \rightarrow A: y$ given $A: x$.) In [i $\bar{b}$, it is stated, but not proven, that the free variable version of the $(\mathrm{PB})$ rule only need to be used at most once for each occurrence of a $(\mathrm{T} \rightarrow)$ or $(\mathrm{F} \otimes)$ signed labelled formula. This restriction together with an examination of the labels of the F-formulas in an LKE-tree, which may allow restrictions on the

9 For example, the third solution corresponds to a tree in which the signed labelled formula $T X$ is used twice, and in which $T(A \rightarrow D) \otimes(D \rightarrow E): c d(c d e / d)$ and then $D \rightarrow E:(c d(c d e / d)) / d$ are derived and then finally $T E:((c d(c d e / d)) / d) d a$ is derived. Using the associativity of $\circ$ and the simplification rule for the / operator, namely $x z(y / z) \sqsubseteq x y$, it can be shown that $(c d e / d) d a \sqsubseteq e c d a$ and $((c d(c d e / d)) / d) d a \sqsubseteq c d(c d e / d) a \sqsubseteq e c c d a$. 
labels of T-formulas derived from applications of $(\mathrm{T} \rightarrow)$, can be used to impose finiteness on the LKE-trees. The second difficulty is concerned with algorithms to solve general inequalities involving the / operator. In the LKE approach, the equivalence (5.1.) given in Section 4.3. may be used to derive some additional general properties, such as $x \circ z \circ(y / x) \sqsubseteq z \circ y$ and $(x / y) / z=x /(y z)$. These properties could be used to solve constraints between terms involving the / operator. Nevertheless, label inequalities still remain difficult to solve. In the ND approach, the difficulty of handling terms with the / operators is avoided by the introduction of imposed constraints. In fact, applications of an imposed constraint in an expansion step of the solving process could be seen as using the general rule $x \circ z \circ(y / x) \sqsubseteq z \circ y$ on an LKE constraint involving the / operator. The search for a limit on the number of expansion steps and control of the use of the (Lemma) rule are instead difficulties for the ND approach as well.

\subsection{Final Remarks}

The method described here provides a way of carrying out natural deduction proofs for the three most well known substructural logics, namely Linear Logic, Relevance Logic and Intuitionistic Logic. To cover the cases of other substructural logics which already exist in the literature or which may be proposed in the future, it is sufficient to appropriately adapt the solving process algorithm, leaving unchanged the set of labelled ND rules given in Table $\underset{2}{2}$ For example, in the case of Lambek calculus the simple instantiation algorithm used for Linear logic can be strengthened to deal with lists rather than multisets in order to avoid the commutativity property. The case of Mingle's implication can be obtained by restricting the monotonicity property to the expansion property (i.e. $x \sqsubseteq x \circ x$ ). This latter case can be dealt with by appropriately adapting the instantiation step of the solving process algorithm for intuitionistic logic

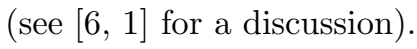

In this paper the description is limited to the syntactical fragment of substructural logics containing only material implication and the multiplicative conjunction. Multiplicative negation can be easily incorporated into the system for classical substructural logics. It would be sufficient to translate negated formulae of the form $\neg A$ into $A \rightarrow \perp$, where $\perp$ is a constant proposition representing falsity, and to introduce the following rule corresponding to proof by contradiction or the double negation rule:

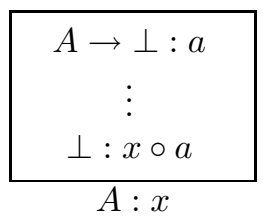

In the presence of negation, $A \otimes B: x$ can equivalently be translated into $\neg(A \rightarrow$ $\neg B): x$. This removes the necessity for the expansion step in the algorithm, leading to guaranteed termination of the solving process for a particular structural derivation and therefore a one to one correspondence of structural derivations with LKE trees (not using $\otimes$ either). The extension to full Linear Logic, including the additive and exponential operators, has been implemented by Kevin Howe [12]". For any of the above extensions as well as for the system described in this paper, the decidability 
property of the system needs still to be proven. This is also the case of the LKE system. It could be foreseen however that to obtain such a result additional controls are needed to restrict (i) the number of (Lemma) rule applications, (ii) the number of $(\rightarrow \mathcal{E})$ rule applications and (iii) the number of expansion steps in the solving process. This is currently under investigation and it is believed that the structural feature of the natural deduction proofs could help in finding such restrictions.

Finally, the issue of uniformity poses one interesting question: What is the price paid for such generality? It is known that the multiplicative fragment of LL (i.e. $\{\otimes, \rightarrow, \neg\})$ is NP-complete. The algorithm described here is EXP-time for the $\{\rightarrow$ $, \otimes\}$ fragment of LL and RL. This appears to be a reasonable complexity, given the theoretical lower bounds. It could be argued that in a theorem prover for LL only, special heuristics could be developed which would improve its efficiency. But this could be done only making the theorem prover specific to one particular logic. In the ND system described in this paper, the modularity of the derivation process given by the use of the labels, would facilitate such heuristics to be embedded into the constraint solving mechanism, leaving the set of ND rules general and applicable to any substructural logic. There is always space then for efficiency gains.

\section{Acknowledgements}

The authors would like to thank Marcello D'Agostino and Hans-Jürgen Ohlbach for their helpful comments and also several anonymous referees for their suggestions for improvements. The authors would also like to acknowledge financial support from the EPSRC and the Brazilian Research Council. Alessandra Russo was initially supported by the EPSRC project GR/J17485 and subsequently by the EPSRC project GR/L55964, whereas Marcelo Finger was partly supported by the Brazilian Research Council (CNPQ), grant 300597/95-9.

\section{References}

[1] K. Broda, Algorithms for Implication LKE, Proceedings of the 1st Workshop on Labelled Deduction (LD'98), 1998.

[2] K. Broda, M. D'Agostino and A. Russo, Transformation methods in LDS, Logic, Language and Reasoning. An Essay in Honor of Dov Gabbay, Kluwer Academic Publishers, 1998.

[3] K. Broda, S. Eisenbach, H. Khoshnevisan, and S. Vickers, Reasoned Programming, Prentice Hall, 1994.

[4] K. Broda and M. Finger, KE-tableaux for a Fragment of Linear Logic, Unpublished Technical Report, 4th Workshop on Theorem Proving with Analytic Tableaux and Related Methods, P. Baumgartner, Ed., University of Koblenz, 1995.

[5] M. D'Agostino, Tableaux Methods for Classical Propositional Logic, Handbook of Tableaux Methods, Kluwer Academic Publishers, M. D’Agostino, D.M. Gabbay, R. Hähnle and J. Possega, Eds., (To appear 1999).

[6] M. D'Agostino and D.M. Gabbay, A generalization of analytic deduction via labelled deductive systems. Part I: Basic Substructural Logics, Journal of Automated Reasoning, 13:243-281, 1994.

[7] M. D'Agostino and M. Mondadori, The taming of the cut, Journal of Logic and Computation, 4:285-319, 1994.

[8] K. Dôsen, A Historical Introduction to Substructural Logics, Substructural Logics, P. Schroeder Heister and K. Dôsen, Eds., 1-31, Oxford University Press, 1993.

[9] D.M. Gabbay, Labelled Deductive Systems, Volume 1 - Foundations, Oxford University Press, 1996. 
[10] G. Gentzen, Unstersuchungen über das logische Schliessen, . Math. Zeitschrift, 39:176-210, 1935, English translation in 18 i].

[11] J.Y. Girard, Linear Logic, Theoretical Computer Science, 50:1-102, 1987.

[12] K. Lee Howe, Abductive Theorem Prover for Substructural Logics, MEng project report, Imperial College of Science, Technology and Medicine, Department of Computing, 1998.

[13] G. E. Mints, Lewis' Systems and System T (1965-1973), Selected Papers in Proof Theory, 221294, Bibliopolis/North-Holand, 1992.

[14] D. Prawitz, Natural deduction : a proof-theoretical study, Almqvist and Wiksell, Stockholm, 1965.

[15] A. Russo, Generalising propositional modal logic using labelled deductive systems, Applied Logic Series (APLS), "Frontiers of Combining Systems, First International Workshop", 3:57-73, 1996.

[16] A. Seiki and J.M. Abe, Natural Deduction and General Annotated Logics, Proceedings of the 1st Workshop on Labelled Deduction (LD'98), 1-14, 1998.

[17] M. Stickel, A Unification Algorithm for Associative-Commutative Functions, Journal of the ACM, 28(3), 1981.

[18] The Collected Papers of Gerhard Gentzen, M. Szabo, Ed., North-Holland, Amsterdam, 1969.

Received 1 August 1996. Revised 13 May 1999 